\title{
1 Aurora B phosphorylates Bub1 to promote spindle assembly checkpoint signaling
}

2 Babhrubahan Roy ${ }^{1}$, Simon J. Y. Han ${ }^{1,2}$, Adrienne N. Fontan ${ }^{1,3}$, Soubhagyalaxmi Jema ${ }^{1}$, Ajit P.

3 Joglekar ${ }^{1, *}$

41 - Cell \& Developmental Biology, University of Michigan Medical School, 109 Zina Pitcher PI.,

5 Ann Arbor, MI-48109, USA

62 - present address: Medical Scientist Training Program, University of Cincinnati College of

7 Medicine, 3230 Eden Ave, Cincinnati, OH 45267, USA

83 - present address: Whitehead Institute for Biomedical Research, Massachusetts Institute of

9 Technology, 455 Main St, Cambridge, MA 02142

* - corresponding author, lead contact: ajitj@umich.edu

11 Twitter handle: @AjitJoglekar1

\section{Summary}

Accurate chromosome segregation during cell division requires amphitelic chromosome attachment to the spindle apparatus. It is ensured by the combined activity of the Spindle Assembly Checkpoint ${ }^{1}$ (SAC), a signaling mechanism that delays anaphase onset in response to unattached chromosomes, and an error correction mechanism that eliminates syntelic attachments $^{2}$. The SAC becomes active when Mps1 kinase sequentially phosphorylates the kinetochore protein Spc105/KNL1 and the signaling proteins that Spc105/KNL1 recruits to facilitate the production of the Mitotic Checkpoint Complex ${ }^{3-8}$ (MCC). The error correction mechanism is regulated by the Aurora B kinase, but Aurora B also promotes SAC signaling via indirect mechanisms ${ }^{9-12}$. Here we present evidence that Aurora B kinase activity directly promotes MCC production by working downstream of Mps1 in budding yeast and human cells. Using the ectopic SAC activation (eSAC) system, we find that the conditional dimerization of Aurora B in budding yeast, and an Aurora B recruitment domain in HeLa cells, with either Bub1 or Mad1, but not the phosphodomain of Spc105/KNL1, leads to ectopic MCC production and mitotic arrest ${ }^{13-16}$. Importantly, Bub1 must recruit both Mad1 and Cdc20 for this ectopic signaling activity. These and other data show that Aurora B cooperates with Bub1 to promote MCC production, but only after Mps1 licenses Bub1 recruitment to the kinetochore. This direct involvement of Aurora B in SAC signaling may maintain SAC signaling even after Mps1 activity

31 in the kinetochore is lowered. 


\section{Results \& Discussion}

34 Dissecting the contributions of Bub1 and Mad1 in Mps1-driven Mitotic Checkpoint 35 Complex (MCC) formation

36 To activate the SAC and delay anaphase onset, unattached kinetochores produce the MCC, 37 which is a complex of four proteins: the 'closed' form of Mad2, Cdc20, BubR1, and Bub3. MCC production is licensed by the Mps1 kinase, which sequentially phosphorylates SAC proteins within the kinetochore to promote their recruitment ${ }^{3-6}$ (Figure 1A). Cdc20 is recruited by Bub1 and BubR1 through constitutive interactions ${ }^{17}$. SAC proteins recruited interact to produce the MCC. Although Mps1 plays the dominant and essential role in this process, Aurora B kinase activity is also required for maximal signaling ${ }^{9-11,18-23}$. In budding yeast, Aurora B phosphorylates Mad3/BubR1 ${ }^{24}$. Whether it phosphorylates other SAC signaling proteins to catalyze MCC production has been difficult to determine, mainly because Mps1 and Aurora B act concurrently within unattached kinetochores. Therefore, to study the effect of Aurora B on SAC proteins, we used the ectopic SAC activation assay or "eSAC"13. In this assay, a mitotic kinase domain is conditionally dimerized with a cytosolic phosphodomain of the kinetochore protein Spc105/KNL1 or Bub1 $1^{13-16}$. The ensuing signaling activity is evident as a mitotic delay.

We first used the eSAC assay to clearly delineate the known Mps1 roles in SAC signaling relying on flow cytometry to quantify the DNA content of yeast cells (Figure 1B). As positive controls, we dimerized Mps1-2xFkbp12 with either 'MELT'-motif containing fragments of the Spc105 phosphodomain or Bub1 or Bub3 fused to Frb and GFP by adding rapamycin to the growth media (Figure 1B). In all cases, cell populations shifted to $2 \mathrm{n}$ ploidy indicating a G2/M arrest $^{25}$ (Figure 1B-C, Figure S1A-B, rapamycin panels in red). Cells lacking Mad2 did not arrest following rapamycin treatment (Figure $1 \mathrm{~B}$, dashed blue panel). Thus, the arrest required a functional SAC.

57 Bub1 contributes two activities for MCC formation. It recruits Mad1 via its central domain, and in

58 human cells, Cdc20 via a conserved 'ABBA' motif 3,5,17,26-28 (Figure 1C). To separate their 59 contributions, we generated bub1 $1^{\text {-abba }}$ wherein the hydrophobic residues predicted to interface 60 with Cdc20 are mutated ${ }^{26}$. Induced dimerization of Mps1 with bub1 $1^{\text {-abba }}$ caused a mitotic arrest 61 suggesting that the ABBA motif is dispensable in Mps1-driven signaling (Figure 1C). 62 Consistently, bub1 $1^{\text {-abba }}$ cells arrested in mitosis following nocodazole treatment (Figure S1C).

63 The fraction of cells escaping the mitotic block was higher compared to wild-type cells indicating 64 that the SAC is weaker (Figure S1C). Mad1-mCherry recruitment to unattached kinetochores in 
bub $1^{\text {-abba }}$ cells was also $~ 50 \%$ lower $^{28}$ (Figure S1D). Bub1-Cdc20 interaction has been difficult to confirm using biochemical methods in HeLa cells ${ }^{29}$. We also could not confirm this interaction in yeast using immunoprecipitation, yeast two-hybrid assays, or microscopy (data not shown). However, the conservation of the ABBA motif sequence supports our assumption that bub $1^{\text {-abba }}$ cannot bind Cdc20.

Mps1 phosphorylates many sites within the Bub1 central domain to promote the Bub1-Mad1 interaction $^{3,5,6,28,30}$. To find the essential phosphorylatable residues, we generated numerous Bub1 mutants wherein subsets of the phosphorylation sites are non-phosphorylatable (Table S1). Some of these mutants weakened, but did not abolish, SAC signaling (Table S1). Mad1 localization to unattached kinetochores was also diminished, but not abolished, even in those mutants that significantly weakened the SAC (Figure S1D). Consistently, Mps1 dimerization with these phosphomutants led to robust eSAC signaling (Table S1). Finally, we dimerized Mps1 with bub1-15A, wherein 15 phosphorylation sites are non-phosphorylatable $\mathrm{e}^{3,16}$. bub1-15A localized to unattached kinetochores in nocodazole-treated cells better than wild-type Bub1. However, it did not activate the either SAC or the eSAC (Figure 1C, Figure S1C and S1E, flow cytometry right panel). Thus, eSAC signaling driven by Mps1-Bub1 dimerization requires Bub1mediated Mad1 recruitment.

In human cells, Mps1 phosphorylates Mad1 to facilitate the formation of Cdc20:C-Mad2 ${ }^{5,6}$. Consistently, induced dimerization of Mps1 with Mad1 arrested yeast cells in mitosis. This arrest was not observed in mad $3 \triangle$ cells, indicating that it was mediated by ectopic SAC signaling (Figure 1D). eSAC activity persisted in spc105-6A cells, wherein all six MELT motifs in Spc105 are non-phosphorylatable, and in bub3 $\triangle$ cells. Thus, this ectopic SAC activation does not require Bub1 localization to the kinetochore. The latter observation also indicates that the budding yeast MCC can be formed ectopically without Bub3 $3^{31}$. Consistent with the dispensability of the Bub1 ABBA motif for Mps1-driven eSAC signaling, Mps1-Mad1 dimerization produced a robust mitotic arrest in bub1 $1^{\text {-abba }}$ cells (Figure 1D). Finally, Mps1 dimerization with mad1-4A, wherein residues implicated in the Mad1-Cdc20 interaction are non-phosphorylatable (Figure 1D), did not affect the cell cycle ${ }^{5,6}$. Interestingly, Mps1-Mad1 dimerization did not affect cell cycle progression in cells expressing bub1-15A likely because Bub1 scaffolding of Mad1 and Cdc20 is necessary for the ectopic MCC assembly ${ }^{32,33}$ (Figure S1F).

MCC formation also requires BubR1, known as Mad3 in budding yeast. In metazoa and fission yeast, BubR1 is recruited to the kinetochore by Bub1 ${ }^{16,34,35}$. However, this Bub1-BubR1 interaction is likely not conserved in budding yeast ${ }^{36}$. To confirm this, we fused GFP to the N- 
terminus of Mad3 (C-terminal fusion of GFP to Mad3 makes it incompetent in SAC signaling, data not shown) and visualized it in nocodazole-treated cells. GFP-Mad3 expressed from the endogenous promoter was undetectable at unattached kinetochores (data not shown). Even when exogenous GFP-Mad3 was significantly overexpressed, it rarely colocalized with unattached kinetochores (Figure 1E and Figure S1G).

These observations suggest the following model for Mps1-driven SAC signaling in budding yeast. As established by previous studies, Mps1 licenses the sequential recruitment of Bub1Bub3 and Mad1-Mad2 by phosphorylating the MELT motifs and Bub1. ABBA motif in Bub1 contributes to SAC signaling, but it is not essential. Mps1 also phosphorylates Mad1 to promote its interaction with Cdc20, and this is essential for MCC formation. Finally, the lack of GFP. Mad3 localization suggests that unattached yeast kinetochores mainly catalyze Cdc20-C-Mad2 formation.

\section{Testing whether Aurora B/Ipl1 kinase activity promotes MCC formation}

The Aurora B kinase, known as Ipl1 in budding yeast, is implicated in SAC signaling. Ipl1 is positioned in unattached kinetochores to act on SAC proteins recruited to the kinetochore by Mps1 activity ${ }^{37}$ (Figure 2A). Therefore, it may also phosphorylate Spc105/KNL1, Bub1, or Mad1 and thus promote MCC formation. To test this hypothesis, we conditionally dimerized Ipl1 with either a fragment of Spc105 spanning its six 'MELT' motifs, Bub1, or Mad1. Rapamycin-induced dimerization of Ipl1 with the Spc105 fragment did not affect the cell cycle, but its dimerization with either Bub1 or Mad1 induced a G2/M arrest (Figure 2B). The morphology of these cells was consistent with a metaphase-arrest: large buds and an intact spindle with two bioriented kinetochore clusters (Figure 2B). Ipl1-Bub1 dimerization did not affect cell cycle progression in mad1 $\Delta$ mutants (Figure 2B blue curve in the middle panel). Thus, the arrest required a functional SAC.

To ensure that Ipl1-Bub1 dimerization did not affect kinetochore biorientation, we created a strain expressing Ipl1 and Ipl1-FRB both. Chromosome IV bioriented efficiently in both control and rapamycin-treated cells of this strain (Figure 2C top, Figure S2A). A kinase-dead allele of Ipl1, Ipl1 ${ }^{\mathrm{K} 133 \mathrm{R}}$, did not cause the G2/M arrest when dimerized with Bub1 indicating that the kinase activity of Ipl1 is required for eSAC signaling ${ }^{38}$ (Figure $2 \mathrm{C}$ top). Finally, to confirm that the mitotic arrest resulting from Ipl1-Bub1 dimerization is independent of kinetochores, we used a fragment of Bub1, bub1 ${ }^{368-609}$, comprising just the central domain, lacking the Bub3-binding 
'GLEBS' domain and the kinase domain ${ }^{39}$. GFP-bub1 $1^{368-609}-2 x F k b p 12$ did not localize to unattached kinetochore clusters in nocodazole-treated cells (Figure 2C bottom). Importantly,

132 Ipl1 dimerization with GFP-bub1 ${ }^{368-609}-2 x F k b p 12$ robustly activated the SAC.

133 To find sites within the Bub1 central domain phosphorylated upon its dimerization with Ipl1, we 134 affinity purified GFP-bub1 ${ }^{368-609}-2 x F k b p 12$ from rapamycin- and DMSO-treated cells (Figure 135 S2B). In SDS-PAGE analysis, the mobility of GFP-bub1 ${ }^{368-609}-2 x F k b p 12$ affinity-purified from rapamycin-treated cells was retarded, suggesting that it was post-translationally modified (Figure S2B). Mass spectrometry identified T438, S474, S475, T550, T556, and S596 in the Bub1 central domain as phosphorylated (Table S2). Importantly, these phosphorylations were significantly enriched in rapamycin-treated cells. Although our analysis identified only one of the 15 sites implicated in Bub1-Mad1 interaction, it suggests that following its rapamycin-induced dimerization, Ipl1 phosphorylates Bub1, and potentially Mad1, to drive eSAC signaling.

Intact ABBA motif in Bub1 is essential for the Aurora B/lpl1-mediated ectopic SAC activation

We next examined the residues in Bub1 needed for eSAC activity. Ipl1 dimerization with bub1 ${ }^{\text {T453A, T455A }}$ did not affect cell cycle progression (Figure 2D top). Ipl1 dimerization with bub1 ${ }^{\text {T453A }}$ (predicted to be a Cdk1 phospho-site) arrested the cell cycle, implicating Bub1(455T) as a residue critical for Ipl1-driven eSAC signaling (Figure S2C left). Bub1(485T) was also similarly found to be critical (Figure 2D top, Figure S2C and S2D). Since Ipl1 and Mps1 can both phosphorylate Bub1, we wanted to know if Mps1 becomes non-essential when Ipl1 is dimerized with Bub1. Ipl1-driven eSAC signaling was abolished when the kinase activity of an analog-

152 sensitive Mps1 allele was conditionally inhibited ${ }^{40}$ (Figure S2E). Thus, Mps1 must prime Bub1 153 for Ipl1 activity.

154 We next tested whether the Bub1 ABBA motif is essential for Ipl1-driven eSAC signaling. 155 Surprisingly, Ipl1 dimerization with bub1 $1^{\text {-abba }}$ did not affect the cell cycle (Figure 2D bottom flow 156 cytometry panel). Mps1-driven eSAC activity is unaffected by the same mutation (Figure 1C). 158 Ipl1 dimerization with Mad1 in either cells lacking Bub1 or in cells that express either bub1 ${ }^{\text {-abba }}$ or bub1 $^{\mathrm{T} 485 \mathrm{~A}, \mathrm{~T} 509 \mathrm{~A}, \mathrm{~T} 18 \mathrm{~A}}$ did not affect the cell cycle (Figure 2E). 
These data advance the following model for the direct role of Ipl1 in MCC production. Mps1 activates the SAC by sequentially phosphorylating the MELT, Bub1, and Mad1 to license the recruitment of Bub1-Bub3, Mad1-Mad2, and Cdc20, respectively. Although Ipl1 localizes to unattached kinetochores, it cannot activate the SAC on its own because it cannot phosphorylate the MELT motifs ${ }^{13,14}$. After Mps1 phosphorylates the MELT motifs and Bub1, Ipl1 phosphorylates Bub1 to further promote Mad1-Mad2 recruitment. Finally, Ipl1 cannot phosphorylate Mad1 to enable the Mad1-Cdc20 interaction; Mad1 must cooperate with Bub1 to promote MCC formation ${ }^{32,33}$.

At this juncture, it is important to note two properties of the eSAC system that likely accentuate it's signaling activity. First, by labelling the genomic copy of Ipl1 with Frb, nearly all Ipl1 in a cell will dimerize with the Fkbp12-tagged SAC protein (depending on its relative abundance). Second, the high affinity of rapamycin-mediated dimerization of Fkbp12 and Frb will allow Ipl1 to maximally phosphorylate the signaling protein. Under physiological conditions, this Ipl1 contribution is likely to be significantly smaller ${ }^{41}$.

\section{Evidence supporting a direct role of Aurora $\mathrm{B} / \mathrm{lpl} 1$ in driving MCC assembly in kinetochore-based SAC signaling}

To detect the contribution of Ipl1 to kinetochore-based SAC signaling, two conditions must prevail. First, Mps1 activity in the kinetochore must be minimal so that the Ipl1 contribution can be detected. Second, Bub1 must still be recruited to the kinetochore so that Ipl1 can act on it. As an appropriate model for these conditions, we used $s p c 105^{R A S A}$, a well-characterized mutant of Spc105. In this mutant, the highly conserved 'RVSF' motif that binds Protein Phosphatase 1 (PP1) is inactivated. Consequently, the MELT motifs remain phosphorylated, and Bub3-Bub1 remains bound to the MELT motifs in bioriented kinetochores ${ }^{14}$. Thus, the SAC remains active even after kinetochore biorientation ${ }^{30,42,43}$. The concurrence of persistent Bub1 recruitment to stably attached kinetochores with diminished Mps1 activity provides the requisite conditions for observing Ipl1 contributions to SAC signaling. Indeed, the study by Rosenberg et al. found that a hypomorphic, temperature-sensitive mutant of Ipl1 suppresses the SAC-mediated lethality of spc105 $5^{\text {RASA }}$.

The Bub1 ABBA motif is necessary for Ipl1 mediated, but not for Mps1-mediated, SAC signaling. Therefore, we studied genetic interactions between bub1 $1^{\text {-abba }}$ and $s p c 105^{R A S A}$. We found that bub1 $1^{\text {-abba }}$ mutation rescued the viability of $s p c 105^{R A S A}$ (Figure 2F, left). We observed a 
192

193

194

195

196

197

198

199

200

201

202

203

204

205

206

207

208

209

210

211

212

213

214

215

216

217

218

219

220

221

222

223

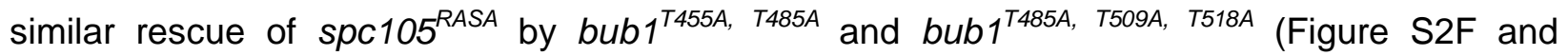
bub1 ${ }^{\text {T453A,T455A, also see reference }}{ }^{30}$ ). Importantly, the bub1 $1^{\text {abba }}$ spc105 ${ }^{\text {RASA }}$ mutant had a functional checkpoint (Figure 2E, bottom). These genetic interactions imply that the Bub1mediated recruitment of both Mad1 and Cdc20 is necessary for persistent SAC signaling from bioriented kinetochores in the spc105 ${ }^{\text {RASA }}$ mutant. Together with the requirement of Ipl1 kinase activity for SAC signaling in the $s p c 105^{R A S A}$ mutant, these data support our hypothesis that Ipl1 directly promotes MCC formation from unattached kinetochores.

Ipl1 may phosphoregulate the Cdc20 recruited by Bub1 to promote SAC signaling. We ruled out this possibility by studying genetic interactions between $s p c 105^{R A S A}$ and several Cdc20 mutants wherein the known or predicted phosphorylation sites in Cdc20 are non-phosphorylatable (data now shown).

\section{Aurora B drives ectopic SAC signaling in human cells}

In human cells, Aurora B promotes SAC signaling indirectly by: (1) creating unattached kinetochores, (2) potentiating Mps1 recruitment to the kinetochore ${ }^{10}$, and (3) inhibiting the phosphatase activity antagonizing $\mathrm{Mps} 1^{9}$. However, in fission yeast, Aurora $\mathrm{B}$ activity is necessary for maintaining mitotic arrest induced by unattached kinetochores ${ }^{21}$. Thus, the creation of unattached kinetochores is not its only role in SAC signaling. The requirement of Aurora B kinase activity for maximal SAC signaling has also been noted ${ }^{20}$. In human and fission yeast cells, Aurora B kinase activity and Bub1 are both required for SAC signaling driven by Mad1 that is artificially tethering to bioriented kinetochores ${ }^{44-47}$, similar to the requirement of Bub1 when Aurora B and Mad1 are dimerized in yeast cells (Figure 2D).

To test if Aurora B can drive eSAC signaling in HeLa cells, we adapted the previously described eSAC system ${ }^{13}$. We created cell lines that constitutively express mNeonGreen-2xFkbp12 fusion of either a KNL1 (Spc105 homolog) phosphodomain containing six MELT motifs (two tandem copies of a fragment spanning three motifs in $\mathrm{KNL} 1^{48}$ ), the central domain of Bub1, or the Cterminal domain of Mad1 (Figure 3A, Methods). In these cells, we conditionally expressed FrbmCherry-INCENP ${ }^{818-918}$. INCENP ${ }^{818-918}$ binds to and activates Aurora $B$ in human cells ${ }^{49}$. As expected, the INCENP fragment was cytosolic, and did not show detectable localization at kinetochores (Figure S3A). We used the cell-to-cell variation in Frb-mCherry-INCENP ${ }^{818-918}$ expression to characterize the dependence of mitotic duration on the eSAC dosage ${ }^{13}$ (Figure 3A-B). 
224 Rapamycin-induced dimerization of Frb-mCherry-INCENP ${ }^{818-918}$ with mNeonGreen-2xFkbp12 225 did not affect the duration of mitosis (Figure 3D), indicating that INCENP ${ }^{818-918}$ over-expression 226 does not affect mitotic duration. INCENP ${ }^{818-918}$ dimerization with the KNL1 fragment containing 227 six MELT motifs also did not affect mitotic progression (Figure 3B, left, Video S1 left panel). 228 Consistently, western blot analysis of whole cell lysates following rapamycin treatment showed 229 that the 'MEIT' motifs (variants of the consensus 'MELT' sequence) in the eSAC 230 phosphodomain were not phosphorylated (Figure 3C, Figure S3B-C). INCENP ${ }^{818-918}$ 231 dimerization with either Bub1 $1^{231-620}$ or Mad1 $1^{479-725}$ resulted in a dose-dependent mitotic delay 232 (Figure 3B, middle and right, Videos S1 middle and right panels). The maximal delay in both 233 cases was similar in magnitude, and comparable to the maximal delay caused by the 234 dimerization of the Mps1 kinase domain with the same protein fragments (Figure 3D; data 235 marked with an asterisk are reproduced from ${ }^{13}$ for comparison). Thus, Aurora $B$ also drives eSAC signaling by phosphorylating either Bub1 or Mad1, or both.

237

238

239

240

241

242

243

244 245

246

247

248

249

250

251

252

253

254

255

256

Aurora B-driven eSAC activity persisted even when it was dimerized with a fragment of Bub1, Bub1 ${ }^{271-620}$, lacking the Bub3-binding GLEBS motif and the N-terminal TPR domain ${ }^{39,50}$. Thus, the eSAC activity is kinetochore-independent (Figure 3E, also see Figure S4A). The same was true for Mps1-driven eSAC activity (Figure 3E left). In fact, the maximal delay was slightly higher and therefore the eSAC activity stronger, presumably because Bub1 $1^{271-620}$ does not sequester Bub3, thus ensuring the full availability of Bub3-BubR1 for MCC formation ${ }^{51}$. eSAC activity also requires an active Aurora $B$ kinase because direct dimerization of the kinase domain of Aurora B (residues 61-344), with the Bub1 phosphodomain did not affect the mitotic progression (Video S2).

Finally, we tested whether the observed mitotic delays are due to ectopic MCC formation. We synchronized HeLa cells expressing INCENP ${ }^{818-918}$ and Bub1 $1^{231-620}$ in G2/M and then released them into media either with or without rapamycin. In each case, we harvested mitotic cells, prepared clarified cell lysates, and immunoprecipitated Cdc20. We performed the same assay for cells wherein Mps1 kinase domain is dimerized with Bub1 ${ }^{271-620}$-mNeonGreen-2xFkbp12. In both cases, increased amounts of Mad2 co-immunoprecipitated with Cdc20 from rapamycintreated cells compared to DMSO-treated cells (Figure S3D). Thus, INCENP ${ }^{818-918}$ dimerization with Bub1 ${ }^{271-620}$ enhances Cdc20:C-Mad2 levels. The increase in Mad2 was greater in the Mps1-driven eSAC system than in the Aurora B driven system likely because Aurora B works downstream from Mps1. 


\section{The ABBA motif of human Bub1 is necessary for the ectopic SAC activation by Aurora B}

258 We next dimerized Bub1 ${ }^{271-522}$, which lacks the ABBA motif, with either the Mps1 kinase domain 259 or INCENP ${ }^{818-918}$. Mps1-driven eSAC activity persisted in this case, but Aurora B-driven eSAC 260 activity was significantly weaker (Figures $3 E-F$ ). These results mirror our findings from budding 261 yeast: Bub1 ABBA motif is dispensable for Mps1-driven eSAC activity, but necessary for Aurora 262 B-driven eSAC activity. This observation also implies that the mitotic delay observed upon Frb263 mCherry-INCENP ${ }^{818-918}$ dimerization with Mad1 ${ }^{479-725}$ will require the Bub1-Mad1 interaction, 264 which in turn requires Mps1 activity. Rapamycin-induced dimerization of INCENP ${ }^{818-918}$ and 265 Mad1 ${ }^{479-725}$ failed to delay cell division in cells treated with the Mps1-inhibitor Reversine (Figure 266 S3E). Thus, Mps1 kinase activity is still required for the eSAC activity induced upon INCENP ${ }^{818-}$ $267{ }^{918}$ dimerization with Mad1 ${ }^{479-725}$.

Aurora B kinase activity promotes MCC assembly during kinetochore-based SAC signaling

271 To detect the contribution of Aurora B to kinetochore-based SAC signaling in HeLa cells, an 272 additional condition must be fulfilled apart from the two mentioned earlier. Aurora B 273 downregulates PP1 and PP2A activity unattached kinetochores, which both antagonize SAC 274 signaling. To negate these SAC antagonists, it becomes necessary to inhibit their activity ${ }^{9}$ 275 following Aurora B inhibition. To enable this, we used the following approach. We released G1/S 276 synchronized HeLa cells expressing H2B-RFP into the cell cycle and treated them with 277 nocodazole to activate the SAC. In these cells, we partially suppressed Mps1 kinase activity to 278 license Bub1-Bub3 recruitment in the unattached kinetochores ${ }^{48}$. We suppressed PP2A 279 recruitment to the kinetochore either by RNAi mediated knockdown of the five B56 isoforms that 280 target PP2A to the kinetochore or by using Calyculin $A^{52}$.

281 As in prior studies, partial inhibition of Mps1 significantly reduced the average duration of the 282 mitotic arrests to $126 \pm 100$ minutes (mean \pm S.D., Figure 4 A, compared to $>1000$ minutes in 283 cells treated with nocodazole alone, data not shown). B56 RNAi increased the duration of the mitotic arrest to $258 \pm 160$ minutes $^{9}$ (mean \pm S.D., Video S3 left). When Aurora B activity was inhibited using the small molecule inhibitor ZM447439 under the same condition, the mitotic arrest was completely abolished (15 \pm 6 minutes, Video S3 right). Calyculin A-treated cells similarly exited mitosis soon after entering it (Figure 4A). These experiments suggest that 
Aurora B kinase activity contributes to SAC signaling directly and independently from its indirect role in retarding SAC silencing.

290 In summary, our data reveal that Aurora B can phosphorylate Bub1 to promote its interaction 291 with Mad1, and thus contribute directly to MCC formation (Figure 4B). Aurora B does not 292 activate the SAC on its own, because it cannot phosphorylate the MELT motifs in Spc105/KNL1. 293 After Mps1 phosphorylates the MELT motifs and Bub1 ${ }^{5,6}$, Aurora B can act on Bub1 to promote 294 the Mad1-Mad2 recruitment. This coordinates the interaction between Mad1-Mad2 and the 295 Cdc20 molecule recruited by the ABBA motif in Bub1, facilitates in the formation of the closed296 Mad2-Cdc20 complex and, ultimately, the MCC $^{32,33}$. The requirement of Mps1 upstream from 297 Aurora B in kinetochore-based SAC signaling and the significant overlap among the 298 phosphorylation targets of the two kinases prevented us from testing this model directly. 299 Nonetheless, it explains prior observations showing that Aurora B cooperates specifically with 300 Bub1 in SAC signaling ${ }^{18}$.

301 Aurora B's role in promoting MCC formation will be physiologically significant, especially when 302 Mps1 activity in the kinetochore is weakened. One such situation occurs in kinetochores with 303 end-on, but syntelic, attachments (Figure 4C). End-on attachments suppress Mps1 activity ${ }^{53-56}$ 304 and weaken Mad1 recruitment via the SAC signaling cascade and possibly through the fibrous 305 corona $^{57}$. In these kinetochores, Aurora B can promote MCC formation, delay anaphase onset, 306 and thereby reduce chromosome missegregation. Indeed, Aurora B and the ABBA motif in Bub1 307 are both essential for SAC signaling in Taxol-treated cells, wherein kinetochores maintain end308 on attachments ${ }^{17,18}$. The direct role of Aurora B in SAC signaling may also contribute to the 309 positive correlation between centromeric tension and SAC signaling. 
311 This work was funded by the 5R35-GM126983 from NIGMS to APJ. We thank Prof. Mara

312 Duncan and her lab (Department of Cell and Developmental Biology, University of Michigan

313 Medical School) for their help. We acknowledge all the Joglekar lab members for their

314 constructive criticism. We specially thank our past lab member Alan A. Goldfarb, who did the

315 initial pilot assay to show that rapamycin induced dimerization of Mps1-FRB with Mad1-Fkbp12

316 arrests the cell cycle. The authors acknowledge that this work would not have been possible

317 without the HeLa cell line, which was developed from Henrietta Lacks' cells taken without

318 compensation or informed consent.

\section{Author Contributions}

321 Conceptualization, Methodology, Writing - Original Draft, Writing - Review \& 322 Editing, Visualization, and Supervision, B.R. and A.P.J.; Formal Analysis 323 and Investigation, B.R., S.J.Y.H, A.F. S.J. and A.P.J.; Software, Resources, and Funding 324 Acquisition, A.P.J.

\section{Declaration of Interests}

327 We declare that no competing interests exist. 


\section{Figure 1 Dissection of the regulatory role of the Mps1 kinase in the SAC signaling} cascade using the 'eSAC' system. (A) A simplified schematic of the SAC signaling cascade in budding yeast. Black arrows represent regulated recruitment of downstream signaling proteins; black lines represent constitutive protein-protein interactions. The 'Mps1' label over an arrow signifies phosphoregulation of the protein recruitment step by Mps1. Gray arrow represents the assembly of sub-complexes into the mitotic checkpoint complex. (B) The ectopic activation of the SAC signaling cascade (simplified on the left hand) by the rapamycin-induced dimerization of Mps1-2xFkpb12 with a cytosolic fragment of Spc105 containing just one MELT motif (GFPSpc105 $120-128-F r b)$. Graphs on the right show the quantitation of cellular DNA content using flow cytometry over 4 hours after the addition of rapamycin to the growth media. Strain genotype is indicated at the top of each graph. Grey, red and dashed blue lines show cytometry results for DMSO-treated, rapamycin-treated wild-type and mad1s cells respectively. Representative micrographs of yeast cells expressing the indicated, fluorescently labeled proteins (right). Notice that the cytosolic GFP-Spc105 $105^{2-128}$-Frb displays faint kinetochore colocalization in rapamycin-

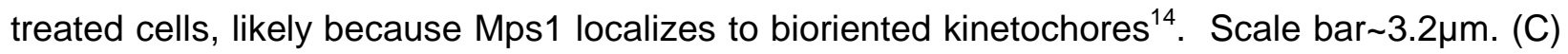
Top: Domain organization of full length Bub1 (Bub1 ${ }^{\mathrm{FL}}$ ) and its middle domain in budding yeast. Bottom left: Schematic of the potential effects of the rapamycin-induced dimerization of Mps1Frb with Bub1-2xFkbp12. Bottom right: Graphs show flow cytometry of DMSO-treated (black lines) and rapamycin-treated (red lines) cells with the indicated genotype. (D) Top: Flow cytometry panels showing effects of rapamycin-induced dimerization of Mps1-Frb with the Mad1-2xFkbp12 in wild-type, in absence of Mad3, in presence of spc105-6A, in absence of Bub3 and in presence of bub1 ${ }^{- \text {abba }}$. Plots with black, red and cyan lines indicate cells treated with

353 DMSO (control), rapamycin and nocodazole respectively. Middle: Domain organization of Mad1-

354 CTD (amino acid 426-749) in budding yeast. Bottom: In left, a partial schematic of SAC cascade

355 is shown. In right the potential effects of the rapamycin-induced dimerization of Mps1-Frb with 356 the Mad1-CTD-2xFkbp12 or Mad1-CTD 4A (T624A, T704A, T737A, T739A)-2xFkbp12 are shown. Color 357 scheme as in B-C. (E) Localization of ectopically expressed GFP-Mad3 in cells arrested in 358 mitosis due to nocodazole treatment. Note that in nocodazole-treated yeast cells typically contain two kinetochore clusters. The larger cluster is proximal to the spindle pole bodies (not visualized), and kinetochores within this cluster are attached to short microtubule stubs. The smaller cluster is distal to the spindle pole bodies (asterisk), and the kinetochores within this cluster are unattached $^{58}$. Scale bars $3.2 \mu \mathrm{m}$. See also Figure S1, Table S1, and Data S1A. 
Figure 2 Rapamycin-induced dimerization of Aurora B/lpl1 with Bub1 and Mad1, but not MELT motifs, leads to ectopic SAC activation. (A) Localization of Ipl1-mCherry and yeast kinetochores (visualized with Spc105 $222:$ :GFP) in yeast cells arrested in mitosis due to nocodazole treatment. Asterisks mark the cluster of unattached kinetochores in each cell. Scale bar $3.2 \mu \mathrm{m}$. (B) Left: Potential effects of the rapamycin-induced dimerization of Ipl1-Frb with the indicated SAC signaling protein. Middle: flow cytometry analysis of DMSO or Rapamycin-treated cultures of indicated strains. Color scheme as in Fig. 1. Right: Micrographs showing morphology of rapamycin-treated cells expressing Ip1-Frb and either Bub1-2XFkbp12 or Mad1-2XFkbp12. Scale bar 3.2 $\mu \mathrm{m}$. (C) Top left: Flow cytometry analysis following rapamycin induced dimerization of Bub1-1xFkbp12 with Ipl1 ${ }^{\mathrm{WT}}$ (red) or ipl1 ${ }^{\mathrm{K} 133 \mathrm{R}}$-Frb-GFP (dashed blue). Top right: Representative micrographs of yeast cells expressing Dsn1-mCherry after treatment with DMSO or rapamycin. Scale bar 3.2 $\mu \mathrm{m}$. Bottom left: Flow cytometry panel showing effects of rapamycin induced dimerization of Ipl1-Frb with GFP-bub1 ${ }^{368-609}-2 x F k b p 12$ (Color scheme: DMSO, grey; WT, red; -abba, dashed blue). Bottom right: Representative micrographs of yeast cells expressing Ndc80-mCherry and GFP-bub1 ${ }^{368-609}-2 x F k b p 12$ after treatment with DMSO,

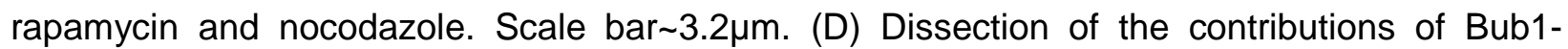
mediated recruitment of Mad1 and Cdc20 in ectopic SAC signaling driven by Mps1 using Bub1 point mutants. 2xFkbp12-tagged Bub1 mutants (indicated at the top of each flow cytometry panel) expressed from the genomic Bub1 locus. (E) Flow cytometric analysis of the effect of rapamycin induced dimerization of Ipl1 and Mad1 in absence of Bub1 (bub1 $\Delta$, top), in presence of bub1 $^{\text {-abba }}$ (middle) and in presence of bub1 ${ }^{\text {T485A, T509A, T518A }}$ (bottom) on the cell cycle. (F) Left: Tetrad dissection of diploids with the indicated genotype. The plate with SPC105/, spc $105^{R A S A}$ were imaged after 2 days (left) and 5 days (middle) before replica plating. The plate with SPC105/A, spc105 ${ }^{R A S A}$, bub1 $1^{\text {-abba }}$ was replica plated after 2 days of incubation and then imaged on the $3^{\text {rd }}$ day. The growth rate of segregants expressing spc105 $s p c 105^{R A S A}$, bub1 ${ }^{\text {-abba }}$ (red circles) is similar to wildtype segregants. The numbers of inviable segregants for $s p c 105 \Delta$, spc105 $5^{\text {RASA }}$, bub1 $1^{\text {-abba }}$ and inviable or micro-colony forming segregants (blue squares) for $s p c 105 \Delta, s p c 105^{R A S A}$ are consistent with the probabilities predicted by the random segregation of three and two genes (0.33 and 0.5$)$ respectively. Right: Flow cytometric analysis of cell cycle progression in nocodazole-treated cells carrying the indicated mutations. See also Figure S2, Table S1, Table S2, Data S1B.

Figure 3 Rapamycin-induced dimerization of INCENP ${ }^{818-918}$ with Bub1 and Mad1, but not with MELT motifs, leads to ectopic SAC activation in HeLa cells. (A) Left: Schematic of the eSAC system designed to test the roles of Aurora B kinase activity in the core SAC signaling 
cascade in HeLa cells. Right: A representative micrograph from a time-lapse experiment showing the variable expression of Frb-mCherry-INCENP ${ }^{818-918}$. Scale bar $~ 8.25$ microns. (B) Schematic at the top displays the domain organization of the eSAC phosphodomain. Scatter plots show the correlation between time in mitosis for a given cell and the average mCherry fluorescence at the beginning of mitosis for that cell. Each gray dot represents one cell $(n=520$, 787, 840 respectively, data pooled from 2 experiments). The blue circles represent the mean of data binned according to the mCherry signal; the horizontal and vertical lines represent the s.e.m. For the Bub1 and Mad1-CTD fragments, the solid blue lines display a four-parameter sigmoidal fit to the binned mean values; $R^{2}$ values $=0.2,0.2$, respectively. (C) Western blot probing for the phosphorylation of the MEIT motif by Aurora B. Also see Figure S3C. (D) Bar graphs display the maximal response predicted by the 4-parameter sigmoidal fit from $\mathrm{B}$. Vertical lines display the $95 \%$ confidence interval for the estimated maximal response. For comparison,

409 the maximal response from eSAC systems comprised of the same three eSAC 410 phosphodomains dimerized with the Mps1 kinase domain is also plotted (data marked by 411 asterisks reproduced from ${ }^{13}$ ). Vertical lines for the M3-M3-Mps1 dimerization represent the 412 standard deviation of the bin corresponding to the peak eSAC response. This representation 413 was made necessary by the non-monotonic nature of the dose-response data. (E) The 414 contributions of the Bub3- and Cdc20-binding domains in Bub1 to the observed eSAC activity 415 driven by either the Mps1 or Aurora B $(n=2635,614$, for the top panel and $n=752,1524$ for 416 the bottom panel; $R^{2}$ values $=0.44,0.24,0.51$, and 0.16 respectively pooled from at least 2 417 experiments). The domain organization of the phosphodomain is displayed in the schematic at 418 the top. Data presented as in B. (F) Comparison of the maximal response elicited from the 419 indicated phosphodomains by either the Mps1 kinase domain or INCENP ${ }^{818-918}$ (predicted mean $420 \pm 95 \%$ confidence intervals). See also Figure S3, Data S1C-E, Video S1 and Video S2.

421 Figure 4 Aurora B contribution to kinetochore-based SAC signaling. (A) Scatter plot 422 displays the duration of the mitotic arrest. Experimental treatments are indicted below each bar. 423 ( $\mathrm{n}=92,48,43,44,41$, experiment performed twice). Cells treated with B56 RNAi and 424 ZM447439 both exited from mitosis very rapidly without assuming the rounded morphology. In 425 this case, entry of the cell into mitosis was visually identified by the release of surface adhesion 426 along with concurrent condensation of Histone H2B signal (in one experiment). Exit from mitosis 427 was identified from the re-spreading off the cell over the surface (micrograph montage at the 428 bottom, also see Video S3). Scale bar 9 microns. (B) Table summarizes the results from the 429 eSAC experiments performed both in yeast and human cells. (C) The proposed mechanism of 430 the direct role of Aurora B kinase activity in SAC signaling. (D) Aurora B-mediated promotion of 
bioRxiv preprint doi: https://doi.org/10.1101/2021.01.05.425459; this version posted October 24, 2021. The copyright holder for this preprint (which was not certified by peer review) is the author/funder. All rights reserved. No reuse allowed without permission.

431 MCC generation may enable kinetochores with syntelic attachments to continue to produce 432 MCC and thus delay anaphase onset. See also Video S3.

433

434

435 


\section{STAR Methods}

437 Resource Availability

438 Lead Contact

439 Further information and requests for resources and reagents should be directed to and will be

440 fulfilled by the Lead Contact, Ajit P. Joglekar (ajitj@umich.edu).

\section{$441 \quad$ Materials Availability}

442 Yeast strains, HeLa cell lines and plasmids constructed in this study are available from the lead 443 contact upon request.

444 Data and Code Availability

- Any additional information required to reanalyze the data reported in this paper is available from the lead contact upon request.

\section{Experimental Model and Subject Details}

\section{Yeast Cell culture}

Yeast strains were grown in YPD (yeast extract $1 \%$, peptone $2 \%$, dextrose $2 \%$ ) or synthetic media supplemented with $2 \%$ dextrose (as per requirement of the yeast strain) at $32^{\circ} \mathrm{C}$. At the time of imaging strains with TetR-GFP and CENIV-TetO, we supplemented the growth media with uracil (final $40 \mu \mathrm{g} / \mathrm{ml}$ ) to distinctively observe TetR-GFP bound to CENIV-TetO array.

To induce meiosis or sporulation, diploid yeast strains were grown in YPD overnight to stationary phase. Next day, the cells were pelleted and resuspended them with starvation media $(0.1 \%$ yeast extract, $1 \%$ potassium acetate, $0.025 \%$ dextrose $)$ and incubated $4-5$ days at RT. To obtain the strain of interest, plates with dissected tetrads were incubated for 2-3 days.

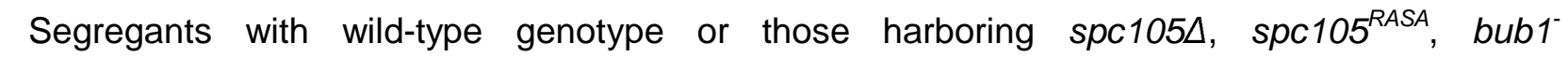
abba/phosphomutants grew within that time period. Infrequently, segregants with $s p c 105 \Delta$, spc $105^{\text {RASA }}$ form micro colonies after 5-6 days of incubation at 30C (Figure 2F, left). To ensure that background mutations are not responsible for the rescue of $s p c 105^{R A S A}$ in bub1 $1^{\text {-abba }}$ background, mutant segregants were back-crossed wild-type parent strains (YEF473). 
463

464

465

466

467

468

469

470

471

472

473

474

475

476

477

478

479

480

481

482

483

484

485

486

487

488

489

490

491

492

For the experiment involving analog sensitive Mps1 yeast cultures were grown for $3 \mathrm{~h}$ till they attained mid-log phase. These cultures were treated with Hydroxyurea (100mM final) for $2 \mathrm{~h}$ $30 \mathrm{~min}$ to synchronize the cells late $S$ phase. Following that, the cells were washed with YPD and released into either YPD (control) or YPD supplemented with 1-NMPP1 (50 $\mu \mathrm{M}$ final). After $15 \mathrm{~min}$ of incubation with 1-NMPP1, either DMSO (control) or rapamycin $(1 \mu \mathrm{g} / \mathrm{ml}$; to mediate the dimerization of Aurora B/Ipl1-Frb-GFP and Bub1-2xFkbp12) was added to the media. Mitotic cells were categorized as prometaphase, metaphase and anaphase cells according to the distribution of fluorescently labeled kinetochores within each cell (representative images in Figure S2E). The unbudded cells were considered as the cells in G1 and thus they were not taken into consideration in our analyses.

\section{Tissue culture and generation of stable cell lines}

Henrietta Lacks (HeLa) cells were grown in DMEM media with 10\% FBS, 1\% Pen/Strep, and 25 $\mathrm{mM}$ HEPES at $37{ }^{\circ} \mathrm{C}$ and $5 \% \mathrm{CO}$. Stable cell lines expressing the two eSAC components were generated by integrating a bi-cistronic eSAC plasmid at an engineered Loxp site in the HeLa genome according to the protocol described in ${ }^{62}$. Clones with stable integration of the eSAC plasmid were selected using Puromycin $(1 \mathrm{ug} / \mathrm{ml})$, and several clones were pooled together to create the cell cultures used in the experiments.

To conduct dose-response analysis, each eSAC cell line was plated $\sim 40-48$ hours prior to the start of the experiment in DMEM media without Puromycin. Doxycycline was added at the time of plating to induce the expression of either Frb-mCherry-Mps1 or Frb-mCherry-INCENP ${ }^{818-918 .}$ Prior to imaging, the cells were washed with PBS. Fluorobrite media with $10 \% \mathrm{FBS}, 1 \%$ Pen/Strep with or without Rapamycin were added to each well.

\section{Method Details}

\section{Plasmid and strain construction for study involving S. cerevisiae}

Plasmids and S. cerevisiae strains and cell lines used in this study are tabulated in Table S3. $S$. cerevisiae strains containing multiple genetic modifications were constructed using standard yeast genetics. Proteins tagged with GFP(S65T) and mCherry or yeast codon optimized mCherry were used to visualize kinetochores, spindle pole bodies and SAC signaling 
components. A 7-amino-acid peptide (sequence: 'RIPGLIN') was used as the linker between the proteins and their C-terminal tags (GFP, mCherry, Frb or 2xFkbp12). The cassettes for gene deletion, gene replacement and $\mathrm{C}$ - terminal tags were introduced at the endogenous locus through homologous recombination of PCR amplicons or using linearized plasmids ${ }^{59}$. In the past, significant strain to strain variation in the intensity of mCherry-tagged kinetochore proteins or checkpoint proteins was observed. This variation arises from the inherent variability of mCherry brightness. Therefore, all Mad1-mCherry strains were created by crossing the same transformant of Mad1-mCherry (AJY1836 or AJY3741) with other strains. The deletion mutant of NUP60 always accompanies Mad1-mCherry to disrupt Mad1localization to the nuclear envelopes ${ }^{60}$. This facilitated clearer imaging and quantification of Mad1 localized to the unattached kinetochores without affecting SAC strength.

To create any diploid yeast strains, overnight cultures of a and a mating types were mixed and spotted on a YPD plate, and then incubated for approximately 3-4 hours at $32^{\circ} \mathrm{C}$.

All the Spc105 mutants used in the study are chimeras of Spc105 and GFP as described previously ${ }^{30,43}$. Genes encoding the chimeric proteins were introduced using a cassette that consists of the $397 \mathrm{bp}$ upstream and $250 \mathrm{bp}$ downstream sequences of the SPC105 open reading frame as promoter ( $p r S P C 105)$ and terminator (trSPC105) sequences respectively. Genes encoding GFP(S65T) at the $222^{\text {nd }}$ or $455^{\text {th }}$ amino acid positions of Spc105 were introduced by sub-cloning with an extra BamHI site (Gly-Ser) upstream and Nhel site (Ala-Ser) downstream from GFP. The plasmids based on pRS305 or pRS306 backbone were linearized by BstEll or Stul before transformations to ensure their integration at the LEU2 or the URA3 locus respectively.

To build bub1 phosphomutants containing plasmids the pSK954 plasmid backbone was used ${ }^{61}$. pSK954 harbors the $A D H 1$ transcription terminator cloned within Ascl-Bg/ll sites. 500bp sequence upstream of the BUB1 start codon, 3.063kb BUB1 ORF sequence harboring the designated mutations and $651 \mathrm{bp} 2 x F K B P 12$ or $705 \mathrm{bp}$ yeast mCherry were subcloned using Sacll-Ascl sites. The ORF and $2 x F K B P 12$ or mCherry were linked by 21 bp linker which codes for RIPGILK. 350bp sequence downstream of the BUB1 stop codon was subcloned between Pmel-Apal to serve as the terminator. To build the strains with bub1 phosphomutant allele, a strain hemizygous for BUB1 was used (AJY6055). The plasmids were digested by Apal and Sacl to release $6.279 \mathrm{~kb}$ fragment which recombined at the deleted bub1 locus replacing the NAT1 cassette. There are two chimeras that express bub1 ${ }^{\text {T453A, T455A }}$ (pAJ852 and pAJ896). BUB1 ORF of pAJ852 harbors mutations of 449SR450::TG. However, upon testing no 
526 phenotypic differences were detected between the strains constructed by pAJ852 and pAJ896.

527 Contrary to Mad1-mCherry strains, bub1-15A-mCherry strains were created via gene

528 replacement using linear fragments (linearized with Apal-Sacl) of pAJ923. Therefore, the

529 difference in intensities between wild-type and bub1-15A observed in figure S1E may have been

530 caused by differences in mCherry brightness rather than protein localization.

531 pSB148 which contains IPL1 ORF flanked $1.0 \mathrm{~Kb}$ promoter (prIPL1) and 654 bp terminator 532 (trIPL1) was acquired from the Biggins lab. FRB-GFP along with ADH1 terminator was first 533 cloned within Agel-Sacl sites to generate a chimera which can express Ipl1-Frb-GFP (pAJ941).

534 Then a 600 bp fragment. which was synthesized by Integrated DNA technologies and harbors 535 kinase dead mutation of Ipl1 (K133R), was cloned within the Swal-Agel sites to build pAJ940. 536 AJY6156 (fpr1D, BUB1-1xFKBP12-HIS3, DSN1-mCherry-HYG) was transformed with Stul 537 digests of these plasmids where they can be integrated in URA3 loci.

538 Similarly, to construct the mutants of putative phosphorylation sites of Cdc20, 506bp sequence 539 upstream of the CDC20 start codon and $1.833 \mathrm{~kb} C D C 20$ ORF sequence harboring the 540 designated mutations were cloned into the Sacll-Ascl sites of pSK954. A Spel site (ACTAGT) 541 was inserted between the promoter and the ORF sequence. Finally, 300bp sequence 542 downstream of the CDC20 stop codon was cloned between Pmel-Kpnl sites. To build strains 543 with cdc20 phosphomutant alleles, a strain hemizygous for CDC20 was used (AJY5249). The 544 plasmids were digested by $K p n l$ and Sacll to release $4.328 \mathrm{~kb}$ fragment which recombined at the CDC20 locus replacing the TRP1 cassette.

\section{Plasmid and cell line construction for study involving HeLa cells}

548 The plasmids used for the stable cell lines were based on the plasmids that have been 549 described previously ${ }^{13}$. Briefly, the phosphodomain was integrated into the constitutively expressed ORF of the plasmid using either Notl or Ascl and Xhol restriction sites. The INCENP ${ }^{818-918}$ fragment was integrated into the conditionally expressed ORF using Fsel and Bg/ll restriction sites.

\section{Flow cytometry}

555 To perform these experiments, the designated strains were grown to mid log phase. Media were 556 then supplemented with Nocodazole (final concentration $15 \mu \mathrm{g} / \mathrm{ml}$ ) to depolymerize the spindle 
557 microtubules and activate the SAC and rapamycin $(1 \mu \mathrm{g} / \mathrm{ml})$ to induce the dimerization of FRB 558 and Fkbp12 fused proteins ${ }^{63}$. Samples containing approximately $0.1 \mathrm{OD}_{600}$ cells were collected $5590,1,2,3$ and $4 \mathrm{~h}$ after nocodazole addition, and the cells were fixed using $75 \%$ ethanol, and 560 stored at $4^{\circ} \mathrm{C}$ overnight. Next day, the cells washed and treated the cells with bovine pancreatic 561 RNase (Millipore Sigma, final concentration $170 \mathrm{ng} / \mu \mathrm{l})$ at $37^{\circ} \mathrm{C}$ for $24 \mathrm{~h}$ in RNase buffer $(10 \mathrm{mM}$ 562 Tris $\mathrm{pH} 8.0,15 \mathrm{mM} \mathrm{NaCl}$ ). Next, RNase was washed out and the cells resuspended in $1 \mathrm{X}$ 563 phosphate buffered saline $(\mathrm{pH} 7.4)$ and stored at $4^{\circ} \mathrm{C}$. These samples were incubated in 564 Propidium lodide (Millipore Sigma, final concentration $5 \mu \mathrm{g} / \mathrm{ml}$ in PBS) for at least $1 \mathrm{~h}$ at RT on 565 the day of the assay. The stained cells were analyzed using the LSR Fortessa (BD Biosciences) 566 in Biomedical research core facility, University of Michigan medical school. For each strain flow 567 cytometry was performed at least twice. Representative results from one of these experiments 568 are displayed in each panel. The data was analyzed using the FlowJO software and the graphs 569 were adjusted by Adobe illustrator. As positive controls for SAC null phenotype, bub1 $\Delta$ or 570 mad2 $\Delta$ strain were used.

\section{Microscopy for S. cerevisiae cells and image analysis}

573 A Nikon Ti-E inverted microscope with a 1.4 NA, 100X, oil-immersion objective was used for all 574 imaging experiments. Additionally, the 1.5X opto-var lens was used to measure Mad1-mCherry 575 intensities. The cells were imaged at room temperature in synthetic dextrose (or synthetic 576 galactose media whenever it was required for the assay) supplemented with essential amino 577 acids to obtain at least 20 microscopic fields at a given time points for any strains. Mounting 578 media were supplemented with nocodazole to image the nocodazole arrested cells. For each 579 field of view, a ten-plane Z-stack was acquired (200nm separation between adjacent planes), 580 and at least 20 fields were acquired in each experiment.

581 Total fluorescence intensities of kinetochore clusters (16 kinetochores in metaphase) were 582 measured by integrating the intensities over a $6 \times 6$ region centered on the maximum intensity 583 pixel. Median intensity of pixels immediately surrounding or a nearby $6 \times 6$ area was used to 584 correct for background fluorescence. Fluorescence intensity was calculated as described 585 previously ${ }^{64,65}$. 
588 To induce the expression of either mCherry-Frb-Mps1 kinase domain or -Incenp ${ }^{818-918}$, 589 doxycycline was added to a final concentration of $2 \mathrm{ug} / \mathrm{ml}$ (stock concentration $2 \mathrm{mg} / \mathrm{ml}$ in 590 DMSO). To induce the dimerization of protein fragments, Rapamycin was added $\sim 1$ hour prior 591 to the start of the experiment to a final concentration of $500 \mathrm{nM}$ (stock concentration $500 \mu \mathrm{M}$ in 592 DMSO). GSK-923295 was added to the final concentration of $15 \mathrm{nM}$ (stock concentration 236 $593 \mu \mathrm{M})$. Partial Mps1 inhibition was achieved by adding Reversine to the final concentration of 250 $594 \mathrm{nM}$ (stock concentration $500 \mu \mathrm{M}$ in DMSO). Nocodazole was added to the final concentration of $595330 \mathrm{nM}$ (stock concentration $330 \mu \mathrm{M}$ in DMSO). ZM447439 was added to the final concentration 596 of $10 \mu \mathrm{M}$ (stock concentration 3mM in DMSO). Calyculin A was added to the final concentration 597 of $100 \mathrm{nM}$ (stock concentration $50 \mu \mathrm{M}$ in DMSO). The cocktail of siRNA against five different B56 598 isoforms was added to a final concentration of $40 \mathrm{nM}$ (stock concentration $10 \mu \mathrm{M}$ ). The siRNA 599 sequences were obtained from ref. ${ }^{9}$.

600

\section{Long term live Cell Imaging of HeLa cells and Image analysis}

602 Imaging was conducted over a period of 24 hours as described in detail previously ${ }^{13}$. Either the 603 Incucyte Zoom Live Cell Imaging system (Sartorus Inc.) or the ImageExpress Nano live cell 604 imaging system (Molecular Devices) using 20x Phase objectives for imaging. To image cells on 605 the Incucyte system, cells were plated in 12-well plastic tissue culture plates, whereas they were 606 plated in 24-well plate glass-bottom dishes for imaging using the ImageExpress Nano system. 607 Typically, 4 positions were selected within each well for imaging. At each position, one phase, 608 GFP, and mCherry image was acquired every 10 minutes. The exposure time for mCherry 609 image was adjusted to minimize photobleaching while ensuring accurate determination of cellular intensity values. It should be noted that the excitation intensity of the Incucyte instrument declined significantly over the course of this study. Furthermore, a small minority of

612 the experiments were carried out on the ImageExpress Nano microscope, which has excitation 613 sources, optics, and detector that are entirely different from the components of the Incucyte 614 microscope. Therefore, the mCherry intensity values across different experiments are not 615 directly comparable. The duration of mitosis and GFP and mCherry fluorescence per cell were 616 determined using a custom image analysis script implemented by a Matlab graphical user 617 interface as described previously ${ }^{13}$. 
620 Immunofluorescence assay was performed as described previously (Deluca et al., 2011, J Cell

621 Sci). We plated cells expressing Frb-mCherry-INCENP ${ }^{818-918}$ on a $12 \mathrm{~mm}$ coverslip until they

622 reached $80 \%$ confluence. The cells were treated with $15 \mathrm{nM}$ GSK-923295 for $3 \mathrm{~h}$ to activate

623 SAC. During fixation, the cells were pre-extracted with $0.5 \%$ Triton X-100 in 0.1 M PHEM (240

$624 \mathrm{mM}$ Pipes, $100 \mathrm{mM}$ HEPES, $8 \mathrm{mM} \mathrm{MgCl} 2$ and $40 \mathrm{mM}$ EGTA) and fixed with 4\% PFA for 10

625 minutes. The coverslips were washed three times with $0.1 \mathrm{M}$ PHEM and blocked for $30 \mathrm{~min}$ at

626 room-temperature with StartingBlock (Thermo scientific, 37578). Next, the cover slips were

627 incubated in primary antibody (Mouse anti-Hec1, 1:3000) over night at $4 \square \mathrm{C}$. Next day, the

628 coverslips were washed 4 times with 1xPHEM containing 0.05\% Tween-20 and incubated with

629 secondary antibodies (Goat anti-Mouse 488, 1: 10000) for an additional $45 \mathrm{~min}$ at room

630 temperature in dark. Following that, the coverslips washed again four times and mounted in

631 antifade (ProLong; Molecular Probes).

632 Immunofluorescence images were taken on a Nikon inverted microscope equipped with a Crest

633 X-Light V2 LFOV25 Spinning Disk Confocal head, and Photometric 95B Prime cMOS camera.

634 For each field of view, $31 \mathrm{Z}$ sections were acquired at $0.2 \mathrm{~mm}$ steps using a $100 \times 3,1.4$

635 Numerical Aperture (NA) Nikon objective.

636

\section{Immunoprecipitation of Cdc20}

638 This assay was performed as described in ${ }^{66}$. Cells expressing Bub1 ${ }^{271-620}$-neon2xFkbp12, FrbmCherry-Mps1 or Bub1 ${ }^{225-620}-\mathrm{mNeon}-2 x F k b p 12$, Frb-mCherry-Incenp ${ }^{818-918}$ were grown to $50 \%$ confluence. These cells were synchronized in S phase using double thymidine treatment

641 (final concentration $2.5 \mathrm{mM}$ ). At the same time, doxycycline (final concentration $2 \mathrm{ug} / \mathrm{ml}$ ) was

642 added to the media to induce expression of Frb-mCherry-Mps1 kinase domain or Frb-mCherry-

643 INCENP ${ }^{818-918}$. G1/S synchronized cells were washed with PBS and released into media 644 supplemented with the Cdk1 inhibitor RO-3306 (final concentration 15 nM). After 20 hours, G2-

645 synchronized cells were washed with PBS and released into media supplemented with 646 rapamycin $(1 \mu \mathrm{g} / \mathrm{ml})$ for $4 \mathrm{~h}$.

647 Mitotic cells were harvested using plate shake-off followed by centrifugation at room 648 temperature/ $1100 \mathrm{rpm} / 5 \mathrm{~min}$. Cells were treated with a volume of lysis buffer (75 mM HEPES, $649 \mathrm{pH} 7.5 ; 150 \mathrm{mM} \mathrm{KCl}, 1.5 \mathrm{mM} \mathrm{MgCl}_{2}, 1.5 \mathrm{mM}$ EGTA, 10\% glycerol, 1\% CHAPS, supplemented 650 with protease inhibitor and phosphatase inhibitor cocktails) added in proportion to the weight of 651 the cell pellet. After a 10 min incubation on ice, total cell lysates were clarified by centrifugation 
652 (4øC/ 13,000 rpm for $10 \mathrm{~min})$. The cell lysates were incubated with a-Cdc20 anitbody653 conjugated protein G-Sepharose 4B beads (4 $\mathrm{gg}$ antibodies per $10 \mu \mathrm{l}$ of beads, Thermo 654 Scientific, catalog \#20399) at room temperature for at least $1 \mathrm{~h}$. After that, the beads were 655 washed three times with lysis buffer and boiled in Laemmli sample buffer.

656

\section{Affinity purification of Bub1 middle domain and mass spectrometry}

658 Mid-log phase cells expressing Ipl1-Frb and GFP-Bub1 ${ }^{368-609}$-2xFkbp12 were treated with either 659 DMSO (control) or Rapamycin for approximately 3 h. GFP-Bub1 ${ }^{368-609}-2 x F k b p 12$ was 660 immunoprecipitated from clarified cell lysates using GFP-TRAP beads following the 661 methodology described previously ${ }^{43,67}$. Precipitated proteins were eluted into Laemmli sample 662 buffer and subjected to SDS-PAGE analysis (See Figure S2B). Incisions $\sim 0.5 \mathrm{~cm}$ tall were made 663 in the Coomassie stained SDS-PAGE, and bands were further processed for tandem mass 664 spectrometry at the University of Michigan proteomics resource facility (See Figure S2B). Mass 665 spectrometry data was filtered to retain peptides/peptide spectrum matches (PSM) with false 666 discovery rate (FDR) $<1 \%$. The detected sites and flanking sequences are included in the table 667 S2. The fold change from DMSO to rapamycin was calculated using normalized peptide 668 abundance.

\section{Immunoblotting}

671 Western blotting was performed using commercial antibodies. The specifications of antibodies 672 and their dilutions are as follows: Mouse $\alpha$-GFP, 1:3,000; mouse $\alpha$-Ds-Red, 1: 2,000; mouse $\alpha$ 673 BTubulin, 1: 15,000; rabbit a-Fkbp12, 1: 5,000; rabbit a-PhosphoMELT (MEIpT, MELT13/17), 1:

674 2000; mouse $\alpha-C d c 20,1: 500$; mouse $\alpha-M a d 2,1: 1000$; mouse $\alpha-B u b R 1,1: 1000$; mouse $\alpha-A p c 3$, 675 1:1000. The primary antibodies were detected using HRP conjugated secondary antibodies (1: 676 10,000) per the manufacturer's instructions. The subsequent chemiluminescence was detected 677 using the C600 imager from Azure Biosystems. The band intensities were measured by 678 Image $^{68}$. 
681

682

683

684

685

686

687

688

689

690

691

692

693

694

695

696

697

698

699

700

701

702

703

704

705

706

707

708

709

710

711

712

Molecular weights of the proteins targeted in the immunoblots and Coomassie stained gels in this study, obtained from Saccharomyces Genome Database and Uniprot: yeast $\alpha$-Tubulin- 49.8 kDa, yeast GFP-Mad1- 115.2 kDa, yeast GFP-Mad3- 87 kDa, yeast GFP-bub1 ${ }^{368-609}$-2xFkbp12$79.2 \mathrm{kDa}$, human Frb-mCherry-INCENP ${ }^{818-918}$ - $50 \mathrm{kDa}$, human $\beta$-Tubulin- $50 \mathrm{kDa}, \mathrm{M6}$ mNeonGreen-2xFkbp12- 101.0 kDa, human Mps1 (Kinase)-Frb-mCherry- 80.0 kDa, human Mad2- 23.5 kDa, human Cdc20- 54.7 kDa, human BubR1- 119.54 kDa, human Apc3- 91.86 kDa.

\section{Quantification and Statistical Analysis}

The technical replicates represent the number of times each experiment was performed. The biological replicates are defined as multiple transformants or segregants of the same strain which contain identical genotype. For imaging experiments, the number of cells analyzed for each strain and number of experimental replications is noted in the figure legends. All statistical analysis was performed using GraphPad Prism (version 8). The data were normalized with the mean intensities obtained for wild-type controls in each experiment to prepare the scatter plots of Mad1 intensities. To compare sample means in all other cases, either the t-test or two-way ANOVA test was applied to ascertain the statistical significance of the rest of the data using GraphPad Prism (version 8). The p-values obtained from these tests are indicated in the figures.

\section{Data S1. Complete images of the immunoblot membranes and gel pictures that were} used in this study. Related to Figure S1G, S2B, 3C, S3A-C. (A) Complete images of the immunoblot membranes probed with anti-aTub and anti-GFP in Figure S1G. Strain details of the cell lysates which were run on the gel are indicated at the top. On the left, the numbers depict the bands of molecular weight marker that was ran along with the lysates. (B) Image of the Coomassie stained whole gel where elutes from GFP-Trap beads were run. Related to Figure S2B. On the left, molecular weight markers are mentioned. (C) Images of the entire immunoblots where lysates of the indicated cell lines were probed with combination of antiphosphoMELT and anti- $\beta$ Tub (left) or with that of anti-DsRed and anti-Fkbp12 (right). Related to Figure $3 \mathrm{C}$ and Figure S3B. Antibody dilutions used in the immunoblots are depicted at the bottom. The numbers on the side of the blots indicate the molecular weight markers. Precise molecular weights of the targeted proteins are also mentioned at the bottom. (D) Images of the complete immunoblots where lysates of the indicated cell lines were probed with combination of 
anti-phosphoMELT and anti- $\beta$ Tub (left) or with that of anti-DsRed and anti-Fkbp12 (right) in Figure S3A. Antibody dilutions used in the immunoblots are depicted at the bottom. The numbers on the side of the blots state the molecular weight markers. Precise molecular weights of the targeted proteins are also stated at the bottom. (E) Images of the immunoblot membranes where input and immunoprecipitated samples after anti-Cdc20 immunoprecipitation experiments were run in Figure S3C. The membrane was cut into three to four strips that were then probed with anti-Cdc20, anti-Mad2, anti-BubR1 and anti-Apc3 antibodies. Precise molecular weights of the targeted proteins are listed on the right of Mps1-eSAC assay immunoblots. The assay was replicated at least thrice for proper quantification and statistical significance.

\section{Supplementary Videos Legend}

Video S1- Effect of rapamycin induced dimerization of Frb-mCherry-INCENP ${ }^{818-918}$ and M3-M3-mNeonGreen-2xFkbp12 (left) or Bub1 ${ }^{231-620}-$ mNeonGreen-2xFkbp12 (middle) or Mad1 ${ }^{479-725}$-mNeonGreen-2xFkbp12 (right) on the duration of mitosis in HeLa A12 cells (hh:mm). Images acquired on the Incucyte microscope. Related to Figure 3B.

Video S2 - Effect of rapamycin induced dimerization of Frb-mCherry- aurora $B^{61-344}$ with Bub1 $^{231-620}$-mNeonGreen-2xFkbp12 on the duration of mitosis in HeLa A12 cells (hh:mm). Images acquired on the Incucyte microscope. Related to Figure 3B.

Video S3 - Left: Cell cycle progression of H2B-RFP expressing HeLa A12 cells treated with $330 \mathrm{nM}$ nocodazole, $250 \mathrm{nM}$ Reversine, and a cocktail of siRNA against five B56 isoforms (hh:mm). Right: Cell cycle progression of H2B-RFP expressing HeLa A12 cells treated as in left along with $10 \mu M$ ZM447439 (hh:mm). Images acquired on the ImageExpress Nano microscope. Related to Figure 4A.

Table S3. list of S. cerevisiae strains and list of plasmids constructed and used in this study. List of yeast strain is related to Figure 1, 2, S1 and S2. Plasmid list is related to Figure 14 as well as Figure S1-S3. Related to Star Method and key resource table. 


\section{References}

743 1. Musacchio, A. (2015). The Molecular Biology of Spindle Assembly Checkpoint Signaling

2. Lampson, M.A., and Cheeseman, I.M. (2011). Sensing centromere tension: Aurora B and the regulation of kinetochore function. Trends Cell Biol 21, 133-140.

3. London, N., and Biggins, S. (2014). Mad1 kinetochore recruitment by Mps1-mediated phosphorylation of Bub1 signals the spindle checkpoint. Genes Dev 28, 140-152.

4. London, N., Ceto, S., Ranish, J.A., and Biggins, S. (2012). Phosphoregulation of Spc105 by Mps1 and PP1 regulates Bub1 localization to kinetochores. Curr Biol 22, 900-906.

5. Ji, Z., Gao, H., Jia, L., Li, B., and Yu, H. (2017). A sequential multi-target Mps1 phosphorylation cascade promotes spindle checkpoint signaling. Elife 6 .

6. Faesen, A.C., Thanasoula, M., Maffini, S., Breit, C., Muller, F., van Gerwen, S., Bange, T., and Musacchio, A. (2017). Basis of catalytic assembly of the mitotic checkpoint complex. Nature.

7. Tipton, A.R., Ji, W., Sturt-Gillespie, B., Bekier, M.E., 2nd, Wang, K., Taylor, W.R., and Liu, S.T. (2013). Monopolar spindle 1 (MPS1) kinase promotes production of closed MAD2 (C-MAD2) conformer and assembly of the mitotic checkpoint complex. J Biol Chem 288, 35149-35158.

8. Hewitt, L., Tighe, A., Santaguida, S., White, A.M., Jones, C.D., Musacchio, A., Green, S., and Taylor, S.S. (2010). Sustained Mps1 activity is required in mitosis to recruit OMad2 to the Mad1-C-Mad2 core complex. J Cell Biol 190, 25-34.

9. Nijenhuis, W., Vallardi, G., Teixeira, A., Kops, G.J., and Saurin, A.T. (2014). Negative feedback at kinetochores underlies a responsive spindle checkpoint signal. Nat Cell Biol 16, 1257-1264.

10. Saurin, A.T., van der Waal, M.S., Medema, R.H., Lens, S.M., and Kops, G.J. (2011). Aurora B potentiates Mps1 activation to ensure rapid checkpoint establishment at the onset of mitosis. Nat Commun 2, 316.

11. Pinsky, B.A., Kung, C., Shokat, K.M., and Biggins, S. (2006). The Ipl1-Aurora protein kinase activates the spindle checkpoint by creating unattached kinetochores. Nat Cell Biol 8, 78-83.

12. Nijenhuis, W., von Castelmur, E., Littler, D., De Marco, V., Tromer, E., Vleugel, M., van Osch, M.H., Snel, B., Perrakis, A., and Kops, G.J. (2013). A TPR domain-containing Nterminal module of MPS1 is required for its kinetochore localization by Aurora B. J Cell Biol 201, 217-231.

13. Chen, C., Whitney, I.P., Banerjee, A., Sacristan, C., Sekhri, P., Kern, D.M., Fontan, A., Kops, G., Tyson, J.J., Cheeseman, I.M., and Joglekar, A.P. (2019). Ectopic Activation of the Spindle Assembly Checkpoint Signaling Cascade Reveals Its Biochemical Design. Curr Biol 29, 104-119 e110.

14. Aravamudhan, P., Goldfarb, A.A., and Joglekar, A.P. (2015). The kinetochore encodes a mechanical switch to disrupt spindle assembly checkpoint signalling. Nat Cell Biol 17, 868-879.

15. Yuan, I., Leontiou, I., Amin, P., May, K.M., Soper Ni Chafraidh, S., Zlamalova, E., and Hardwick, K.G. (2017). Generation of a Spindle Checkpoint Arrest from Synthetic Signaling Assemblies. Curr Biol 27, 137-143.

16. Leontiou, I., London, N., May, K.M., Ma, Y., Grzesiak, L., Medina-Pritchard, B., Amin, P., Jeyaprakash, A.A., Biggins, S., and Hardwick, K.G. (2019). The Bub1-TPR Domain Interacts Directly with Mad3 to Generate Robust Spindle Checkpoint Arrest. Curr Biol 29, 2407-2414 e2407. 
826

827

828

829

830

831

832

833

834

835

836

837

838

17. Di Fiore, B., Davey, Norman E., Hagting, A., Izawa, D., Mansfeld, J., Gibson, Toby J., and Pines, J. (2015). The ABBA Motif Binds APC/C Activators and Is Shared by APC/C Substrates and Regulators. Developmental Cell 32, 358-372.

18. Morrow, C.J., Tighe, A., Johnson, V.L., Scott, M.I., Ditchfield, C., and Taylor, S.S. (2005). Bub1 and aurora B cooperate to maintain BubR1-mediated inhibition of APC/CCdc20. J Cell Sci 118, 3639-3652.

19. Ditchfield, C., Johnson, V.L., Tighe, A., Ellston, R., Haworth, C., Johnson, T., Mortlock, A., Keen, N., and Taylor, S.S. (2003). Aurora B couples chromosome alignment with anaphase by targeting BubR1, Mad2, and Cenp-E to kinetochores. J Cell Biol 161, 267280.

20. Santaguida, S., Vernieri, C., Villa, F., Ciliberto, A., and Musacchio, A. (2011). Evidence that Aurora $B$ is implicated in spindle checkpoint signalling independently of error correction. EMBO J 30, 1508-1519.

21. Vanoosthuyse, V., and Hardwick, K.G. (2009). A novel protein phosphatase 1dependent spindle checkpoint silencing mechanism. Curr Biol 19, 1176-1181.

22. Hauf, S., Cole, R.W., LaTerra, S., Zimmer, C., Schnapp, G., Walter, R., Heckel, A., van Meel, J., Rieder, C.L., and Peters, J.M. (2003). The small molecule Hesperadin reveals a role for Aurora $B$ in correcting kinetochore-microtubule attachment and in maintaining the spindle assembly checkpoint. J Cell Biol 161, 281-294.

23. Vader, G., Cruijsen, C.W., van Harn, T., Vromans, M.J., Medema, R.H., and Lens, S.M. (2007). The chromosomal passenger complex controls spindle checkpoint function independent from its role in correcting microtubule kinetochore interactions. Mol Biol Cell 18, 4553-4564.

24. King, E.M., Rachidi, N., Morrice, N., Hardwick, K.G., and Stark, M.J. (2007). Ipl1pdependent phosphorylation of Mad3p is required for the spindle checkpoint response to lack of tension at kinetochores. Genes Dev 21, 1163-1168.

25. Haruki, H., Nishikawa, J., and Laemmli, U.K. (2008). The anchor-away technique: rapid, conditional establishment of yeast mutant phenotypes. Mol Cell 31, 925-932.

26. Kim, T., Moyle, M.W., Lara-Gonzalez, P., De Groot, C., Oegema, K., and Desai, A. (2015). Kinetochore-localized BUB-1/BUB-3 complex promotes anaphase onset in C. elegans. J Cell Biol 209, 507-517.

27. Weir, J.R., Faesen, A.C., Klare, K., Petrovic, A., Basilico, F., Fischbock, J., Pentakota, S., Keller, J., Pesenti, M.E., Pan, D., et al. (2016). Insights from biochemical reconstitution into the architecture of human kinetochores. Nature 537, 249-253.

28. Fischer, E.S., Yu, C.W.H., Bellini, D., McLaughlin, S.H., Orr, C.M., Wagner, A., Freund, S.M.V., and Barford, D. (2021). Molecular mechanism of Mad1 kinetochore targeting by phosphorylated Bub1. EMBO Rep, e52242.

29. Vleugel, M., Hoek, T.A., Tromer, E., Sliedrecht, T., Groenewold, V., Omerzu, M., and Kops, G.J. (2015). Dissecting the roles of human BUB1 in the spindle assembly checkpoint. J Cell Sci 128, 2975-2982.

30. Roy, B., Han, S.J.Y., Fontan, A.N., and Joglekar, A.P. (2020). The copy-number and varied strength of MELT motifs in Spc105 balance the strength and responsiveness of the Spindle Assembly Checkpoint. Elife 9.

31. Primorac, I., Weir, J.R., Chiroli, E., Gross, F., Hoffmann, I., van Gerwen, S., Ciliberto, A., and Musacchio, A. (2013). Bub3 reads phosphorylated MELT repeats to promote spindle assembly checkpoint signaling. Elife 2, e01030.

32. Piano, V., Alex, A., Stege, P., Maffini, S., Stoppiello, G.A., Huis In 't Veld, P.J., Vetter, I.R., and Musacchio, A. (2021). CDC20 assists its catalytic incorporation in the mitotic checkpoint complex. Science 371, 67-71. 
872

873

874

875

876

877

878

879

880

881

882

883

884

885

886

887

888

889

33. Lara-Gonzalez, P., Kim, T., Oegema, K., Corbett, K., and Desai, A. (2021). A tripartite mechanism catalyzes Mad2-Cdc20 assembly at unattached kinetochores. Science 371, 64-67.

34. Overlack, K., Primorac, I., Vleugel, M., Krenn, V., Maffini, S., Hoffmann, I., Kops, G.J., and Musacchio, A. (2015). A molecular basis for the differential roles of Bub1 and BubR1 in the spindle assembly checkpoint. Elife 4, e05269.

35. Zhang, G., Mendez, B.L., Sedgwick, G.G., and Nilsson, J. (2016). Two functionally distinct kinetochore pools of BubR1 ensure accurate chromosome segregation. Nat Commun 7, 12256.

36. Tromer, E., Bade, D., Snel, B., and Kops, G.J. (2016). Phylogenomics-guided discovery of a novel conserved cassette of short linear motifs in BubR1 essential for the spindle checkpoint. Open Biol 6.

37. Garcia-Rodriguez, L.J., Kasciukovic, T., Denninger, V., and Tanaka, T.U. (2019). Aurora B-INCENP Localization at Centromeres/Inner Kinetochores Is Required for Chromosome Bi-orientation in Budding Yeast. Curr Biol 29, 1536-1544 e1534.

38. Nakajima, Y., Cormier, A., Tyers, R.G., Pigula, A., Peng, Y., Drubin, D.G., and Barnes, G. (2011). Ipl1/Aurora-dependent phosphorylation of Sli15/INCENP regulates CPCspindle interaction to ensure proper microtubule dynamics. J Cell Biol 194, 137-153.

39. Larsen, N.A., Al-Bassam, J., Wei, R.R., and Harrison, S.C. (2007). Structural analysis of Bub3 interactions in the mitotic spindle checkpoint. Proc Natl Acad Sci USA 104, 12011206.

40. Maure, J.F., Kitamura, E., and Tanaka, T.U. (2007). Mps1 kinase promotes sisterkinetochore bi-orientation by a tension-dependent mechanism. Curr Biol 17, 2175-2182.

41. Biggins, S., and Murray, A.W. (2001). The budding yeast protein kinase Ipl1/Aurora allows the absence of tension to activate the spindle checkpoint. Genes Dev 15, 31183129.

42. Rosenberg, J.S., Cross, F.R., and Funabiki, H. (2011). KNL1/Spc105 recruits PP1 to silence the spindle assembly checkpoint. Curr Biol 21, 942-947.

43. Roy, B., Verma, V., Sim, J., Fontan, A., and Joglekar, A.P. (2019). Delineating the contribution of Spc105-bound PP1 to spindle checkpoint silencing and kinetochore microtubule attachment regulation. J Cell Biol.

44. Ballister, E.R., Riegman, M., and Lampson, M.A. (2014). Recruitment of Mad1 to metaphase kinetochores is sufficient to reactivate the mitotic checkpoint. J Cell Biol 204, 901-908.

45. Maldonado, M., and Kapoor, T.M. (2011). Constitutive Mad1 targeting to kinetochores uncouples checkpoint signalling from chromosome biorientation. Nat Cell Biol 13, 475482.

46. Kuijt, T.E., Omerzu, M., Saurin, A.T., and Kops, G.J. (2014). Conditional targeting of MAD1 to kinetochores is sufficient to reactivate the spindle assembly checkpoint in metaphase. Chromosoma 123, 471-480.

47. Kruse, T., Larsen, M.S.Y., Sedgwick, G.G., Sigurdsson, J.O., Streicher, W., Olsen, J.V., and Nilsson, J. (2014). A direct role of Mad1 in the spindle assembly checkpoint beyond Mad2 kinetochore recruitment. EMBO reports 15, 282-290.

48. Vleugel, M., Omerzu, M., Groenewold, V., Hadders, M.A., Lens, S.M., and Kops, G.J. (2015). Sequential multisite phospho-regulation of KNL1-BUB3 interfaces at mitotic kinetochores. Mol Cell 57, 824-835.

49. Sessa, F., Mapelli, M., Ciferri, C., Tarricone, C., Areces, L.B., Schneider, T.R., Stukenberg, P.T., and Musacchio, A. (2005). Mechanism of Aurora B activation by INCENP and inhibition by hesperadin. Mol Cell 18, 379-391.

50. Lee, S., Thebault, P., Freschi, L., Beaufils, S., Blundell, T.L., Landry, C.R., BolanosGarcia, V.M., and Elowe, S. (2012). Characterization of spindle checkpoint kinase Mps1 
reveals domain with functional and structural similarities to tetratricopeptide repeat motifs of Bub1 and BubR1 checkpoint kinases. J Biol Chem 287, 5988-6001.

51. Mora-Santos, M.D., Hervas-Aguilar, A., Sewart, K., Lancaster, T.C., Meadows, J.C., and Millar, J.B. (2016). Bub3-Bub1 Binding to Spc7/KNL1 Toggles the Spindle Checkpoint Switch by Licensing the Interaction of Bub1 with Mad1-Mad2. Curr Biol 26, 2642-2650.

52. Ishihara, H., Martin, B.L., Brautigan, D.L., Karaki, H., Ozaki, H., Kato, Y., Fusetani, N., Watabe, S., Hashimoto, K., Uemura, D., and et al. (1989). Calyculin A and okadaic acid: inhibitors of protein phosphatase activity. Biochem. Biophys. Res. Commun. 159, 871877.

53. Etemad, B., Kuijt, T.E., and Kops, G.J. (2015). Kinetochore-microtubule attachment is sufficient to satisfy the human spindle assembly checkpoint. Nat Commun 6, 8987.

54. Tauchman, E.C., Boehm, F.J., and DeLuca, J.G. (2015). Stable kinetochore-microtubule attachment is sufficient to silence the spindle assembly checkpoint in human cells. Nat Commun 6, 10036.

55. Kuhn, J., and Dumont, S. (2019). Mammalian kinetochores count attached microtubules in a sensitive and switch-like manner. J Cell Biol 218, 3583-3596.

56. Dick, A.E., and Gerlich, D.W. (2013). Kinetic framework of spindle assembly checkpoint signalling. Nat Cell Biol 15, 1370-1377.

57. Kops, G., and Gassmann, R. (2020). Crowning the Kinetochore: The Fibrous Corona in Chromosome Segregation. Trends Cell Biol 30, 653-667.

58. Aravamudhan, P., Chen, R., Roy, B., Sim, J., and Joglekar, A.P. (2016). Dual mechanisms regulate the recruitment of spindle assembly checkpoint proteins to the budding yeast kinetochore. Mol Biol Cell 27, 3405-3417.

59. Jürg Bähler, Jian-Qiu Wu, Mark S. Longtine, Nirav G. Shah, Amos Mckenzie III, Alexander B. Steever, Achim Wach, Peter Philippsen, and Pringle, J.R. (1998). Heterologous modules for efficient and versatile PCR-based gene targeting in Schizosaccharomyces pombe. YEAST 14, 943-951.

60. Scott, R.J., Lusk, C.P., Dilworth, D.J., Aitchison, J.D., and Wozniak, R.W. (2005). Interactions between Mad1p and the nuclear transport machinery in the yeast Saccharomyces cerevisiae. Mol Biol Cell 16, 4362-4374.

61. Kemmler, S., Stach, M., Knapp, M., Ortiz, J., Pfannstiel, J., Ruppert, T., and Lechner, J. (2009). Mimicking Ndc80 phosphorylation triggers spindle assembly checkpoint signalling. EMBO J 28, 1099-1110.

62. Khandelia, P., Yap, K., and Makeyev, E.V. (2011). Streamlined platform for short hairpin RNA interference and transgenesis in cultured mammalian cells. Proc Natl Acad Sci USA 108, 12799-12804.

63. Gillett, E.S., Espelin, C.W., and Sorger, P.K. (2004). Spindle checkpoint proteins and chromosome-microtubule attachment in budding yeast. The Journal of Cell Biology 164, 535-546.

64. Aravamudhan, P., Felzer-Kim, I., Gurunathan, K., and Joglekar, A.P. (2014). Assembling the protein architecture of the budding yeast kinetochore-microtubule attachment using FRET. Curr Biol 24, 1437-1446.

65. Joglekar, A., Chen, R., and Lawrimore, J. (2013). A Sensitized Emission Based Calibration of FRET Efficiency for Probing the Architecture of Macromolecular Machines. Cell Mol Bioeng 6, 369-382.

66. Collin, P., Nashchekina, O., Walker, R., and Pines, J. (2013). The spindle assembly checkpoint works like a rheostat rather than a toggle switch. Nat Cell Biol 15, 1378-1385.

67. Gupta, A., Evans, R.K., Koch, L.B., Littleton, A.J., and Biggins, S. (2018). Purification of kinetochores from the budding yeast Saccharomyces cerevisiae. Methods in cell biology 144, 349-370. 
bioRxiv preprint doi: https://doi.org/10.1101/2021.01.05.425459; this version posted October 24, 2021. The copyright holder for this preprint (which was not certified by peer review) is the author/funder. All rights reserved. No reuse allowed without permission.

940

941

942

943
68. Schindelin, J., Arganda-Carreras, I., Frise, E., Kaynig, V., Longair, M., Pietzsch, T., Preibisch, S., Rueden, C., Saalfeld, S., Schmid, B., et al. (2012). Fiji: an open-source platform for biological-image analysis. Nat Methods 9, 676-682. 
A $\quad$ Mps1 MELT

$\stackrel{\text { Mps } 1}{\longrightarrow}$
MELPT

Bub3-Bub1 - Cdc20

Bub3-pBub1

I' 1 ad1-Mad2

Mps1 $\downarrow$

Cdc20 — pMad1-Mad2
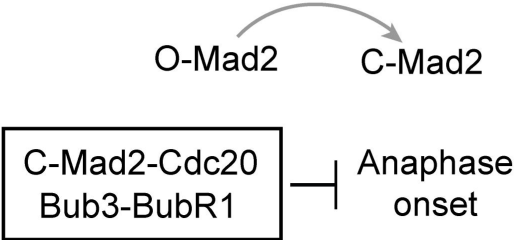

Mitotic

Checkpoint

Complex
Mps1-2xFkbp12

+GFP-Spc105 ${ }^{120-128}$-FRB

Mps1-2xFkbp12

+GFP-Spc105 ${ }^{120-128}$-FRB

Bub3-Bub1

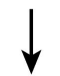

Mad1-Mad2

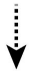

Mitotic

Checkpoint

Complex

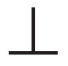

Anaphase

onset

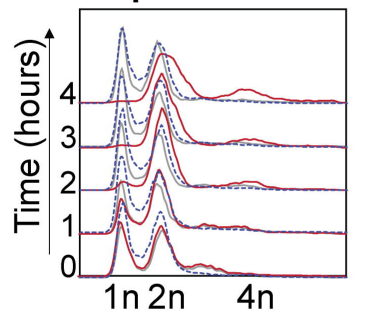

DMSO

WT, rapamycin

mad2s, rapamycin

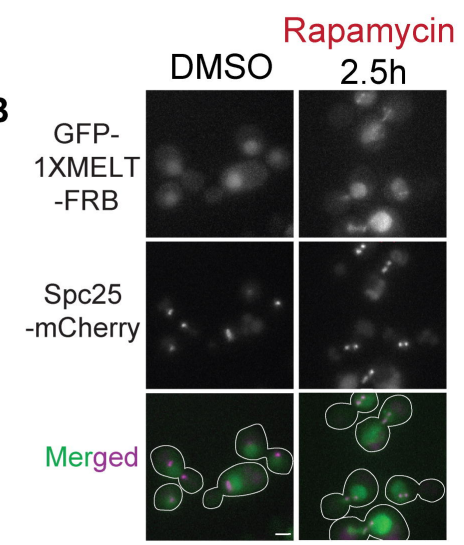

D

C

Bub1

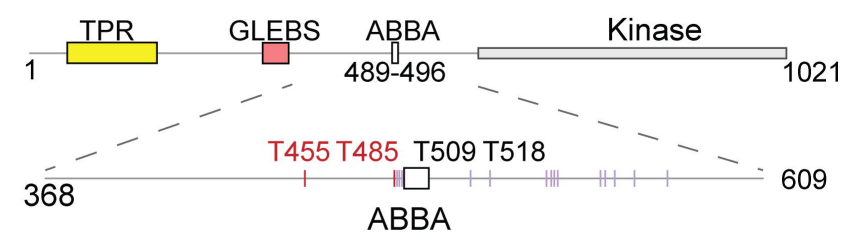

Mps1-Frb +Bub1-2xFkbp12

Bub1 ${ }^{\mathrm{Cdc} 20}$ $\downarrow$ Mad3/BubR1 Mad1-Mad2

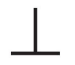

Anaphase onset?

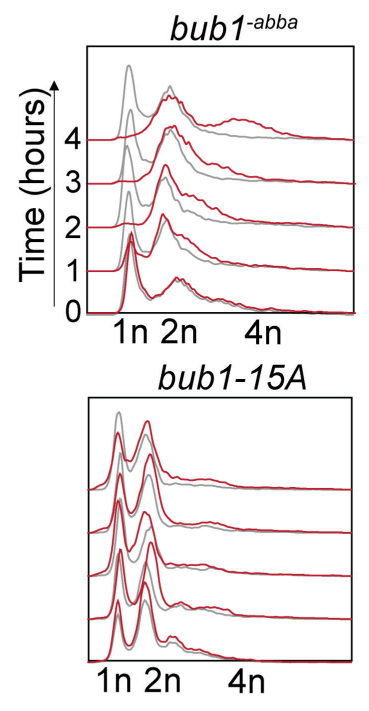

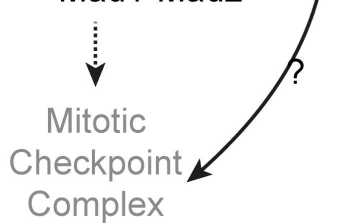

DMSO

Rapamycin 021 09 Mps1-Frb
+Mad1-2xFkbp12 Mad1-Mad2

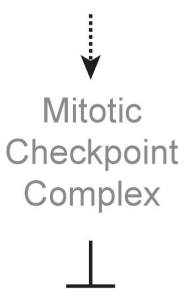

Anaphase onset?
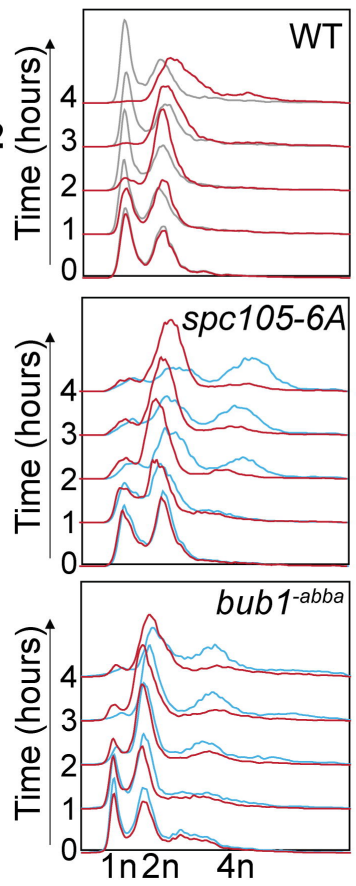

Mad1-CTD

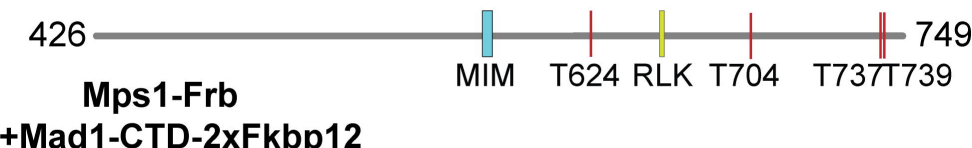

GFP Spc25

-Mad3 ${ }^{\circ}$-mCherry Merged

No

colocalization
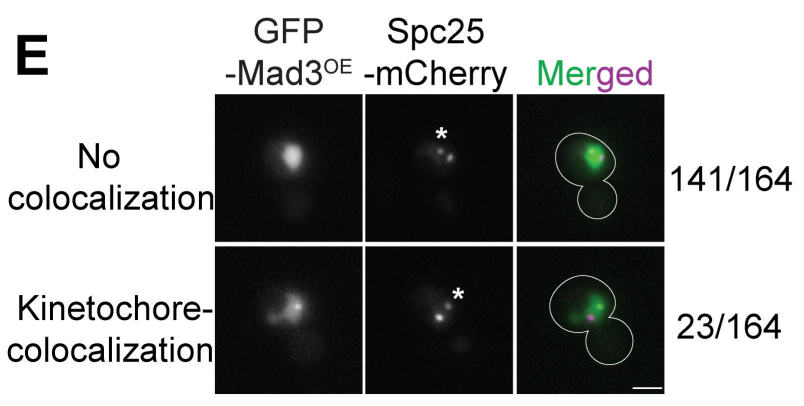

$141 / 164$

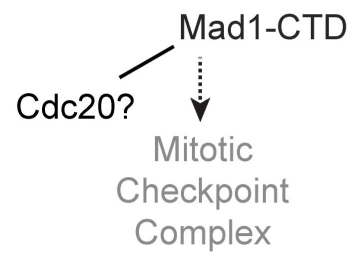

$23 / 164$
Anaphase

onset?
DMSO

Rapamycin

Nocodazole

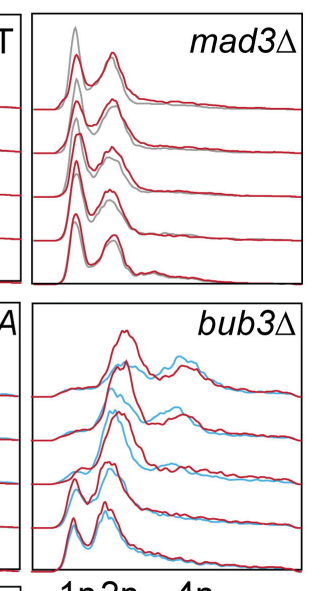

$1 n 2 n \quad 4 n$

49

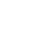
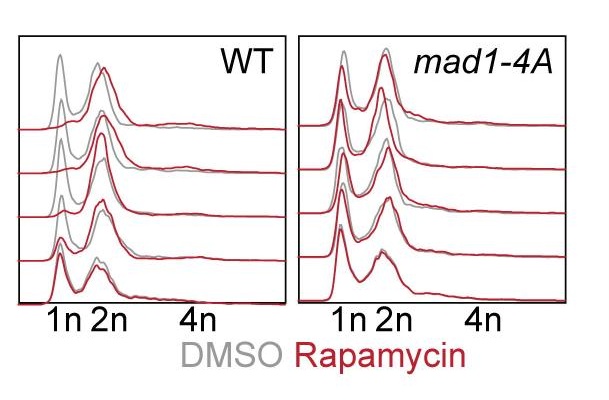


\section{$\mathbf{A}$} Spc105222::GFP
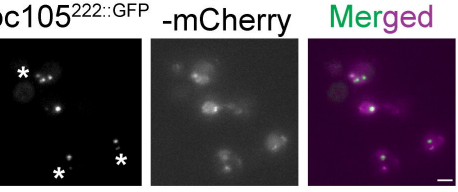

Bright field

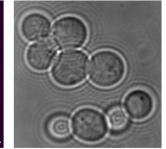

$330 / 467$

B

Ipl1-2xFkbp12

+Spc105 ${ }^{120-329}$-Frb

Ipl1-Frb

+Mad1-2xFkbp12

Ipl1-Frb

+Bub1-2xFkbp12

+Bub1-2xFkbp12

D
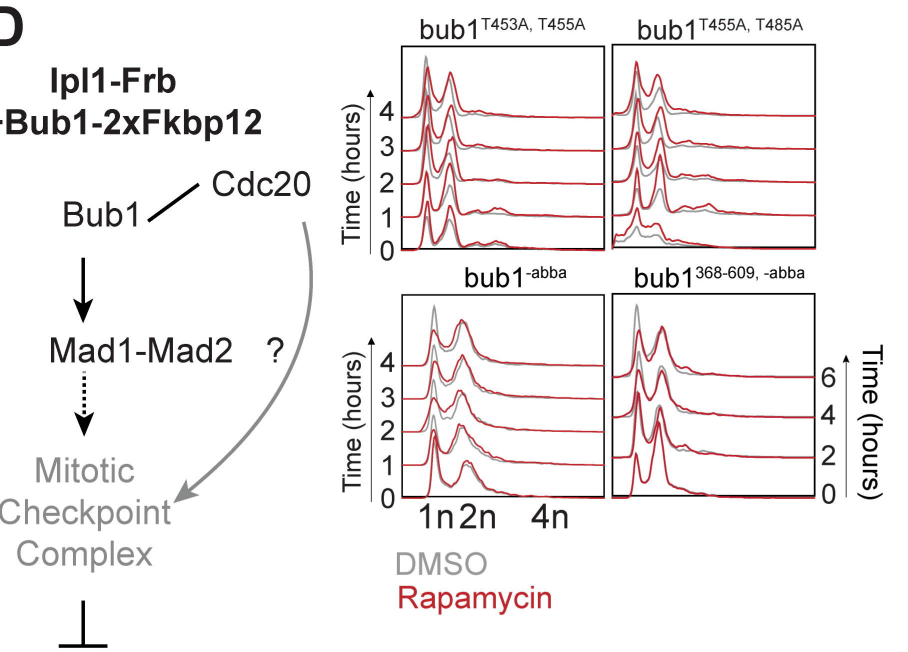

Anaphase

onset?

$\mathbf{F}$

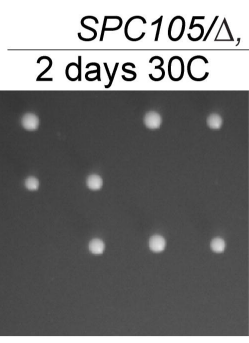

Total tetrads dissected:
DMSO
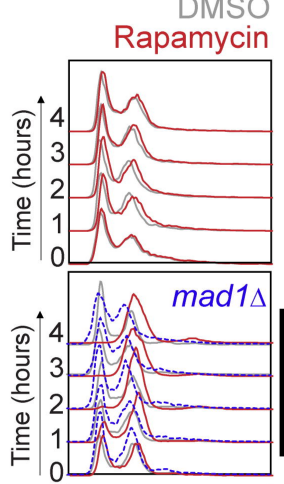

Rapamycin (2.5h)
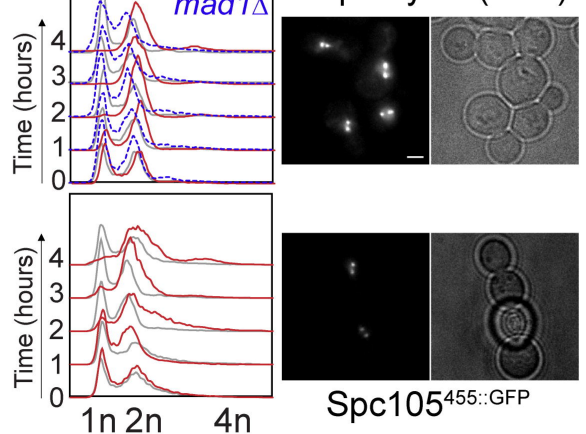

Spc105 $155::$ GFP

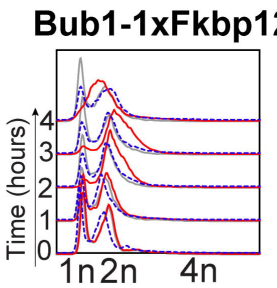

DMSO

Ipl1 ${ }^{\text {WT }}$-Frb, rapamycin ipl1 ${ }^{\mathrm{K} 133 \mathrm{R}}-\mathrm{Frb}$, rapamycin

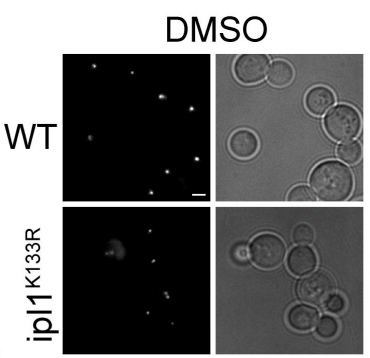

Dsn1-mCherry
Rapamycin (2.5h)

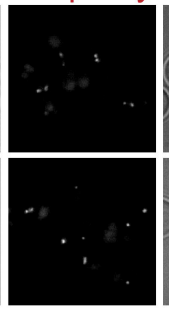

Dsn1-mCherry

GFP

-bub1368-609 Ndc80

-2xFkbp12 -mCherry

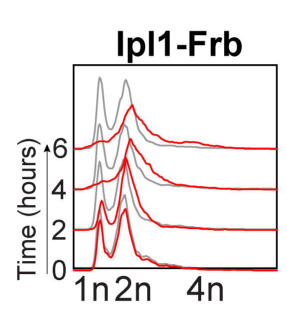

DMSO

Rapamycin
Merged Bright field
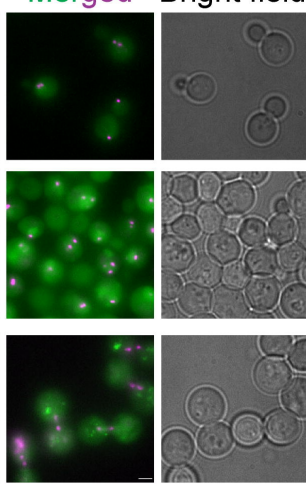

E

bub1s
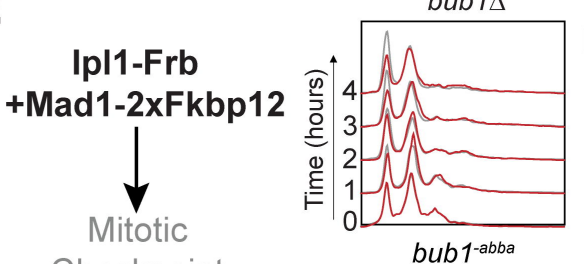

DMSO

Rapamycin
Checkpoint

Complex

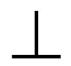

Anaphase

onset?

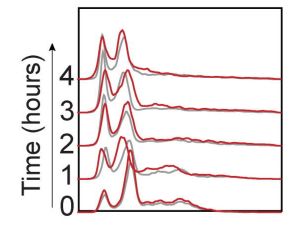

bub1 1485A, T509A, T518A

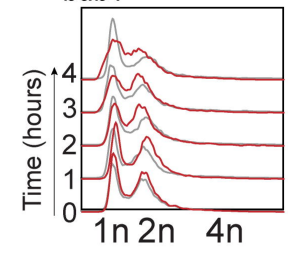

spc105 $105^{\text {ASA }}$

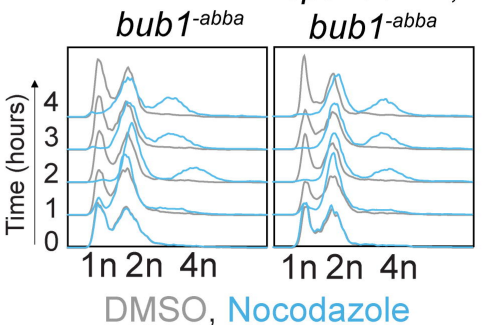

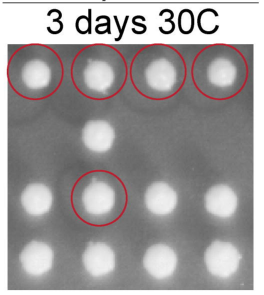

56

Segregants with normal growth:

Segregants with slow growth: 14 Invaible segregants: $\quad 31$ 

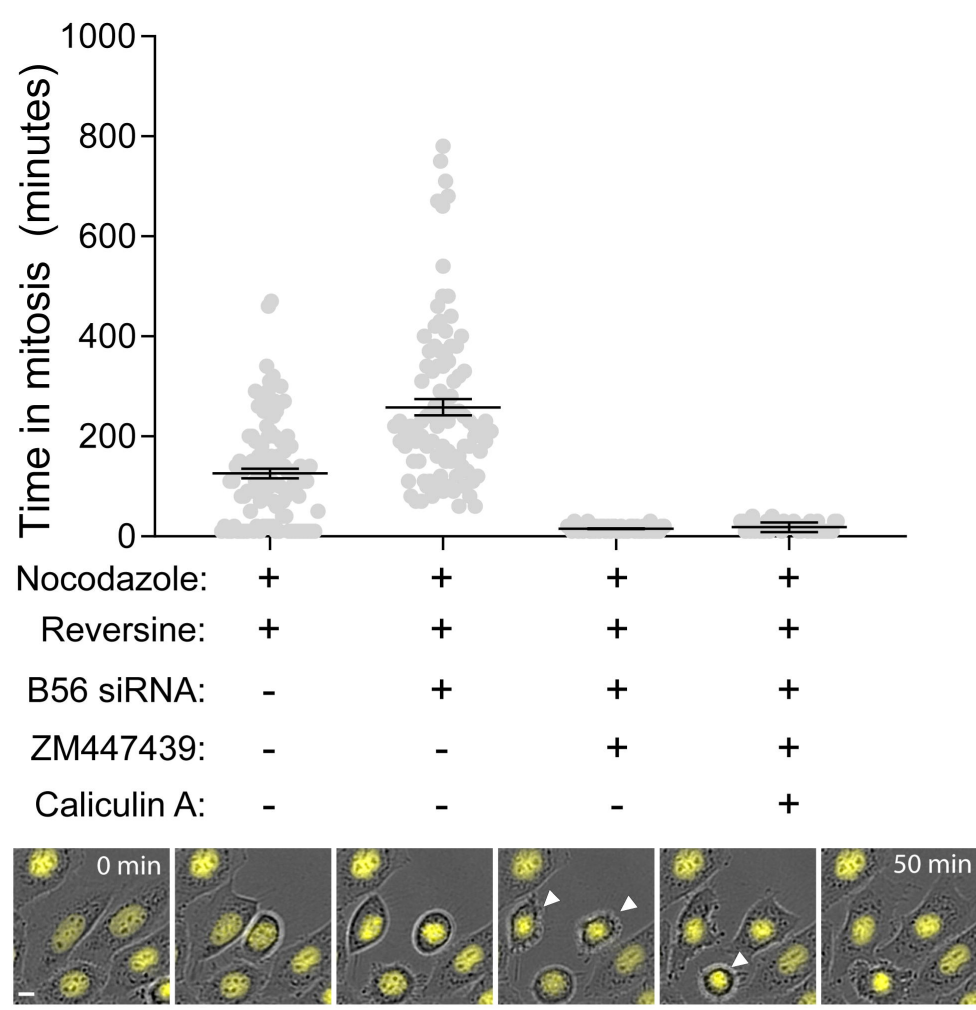

B

\begin{tabular}{cccc}
\hline $\begin{array}{c}\text { Checkpoint } \\
\text { proteins used in } \\
\text { eSAC experiments }\end{array}$ & $\begin{array}{c}\text { Cassettes used in } \\
\text { eSAC experiments }\end{array}$ & $\begin{array}{c}\text { Mps1 mediated } \\
\text { mitotic arrest }\end{array}$ & $\begin{array}{c}\text { Ipl1 mediated } \\
\text { mitotic arrest }\end{array}$ \\
\hline Spc105 & phosphodomain & Yes & No \\
\hline \multirow{2}{*}{ Bub1 } & WT & Yes & Yes \\
& $-A B B A$ & Yes & No \\
Mad1 & - Mad1 & No & No \\
\hline & WT & Yes & Yes \\
& - Cdc20 & No & Not done \\
\hline
\end{tabular}
MELT $\underset{\text { Plk1 }}{\stackrel{\text { Mps1 }}{\longrightarrow}}$ MELTP — Bub3-Bub1 $\stackrel{\text { Plk1 }}{\longrightarrow}$ Cdc20
Mps1 $\downarrow$ Aurora B

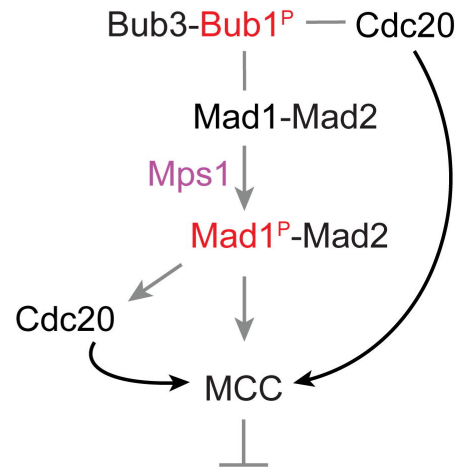

Anaphase

onset
D

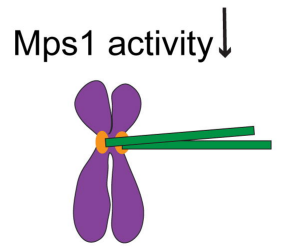

Aurora B activity $\uparrow$

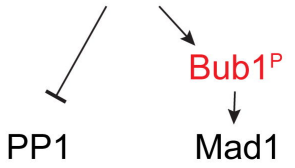

recruitment recruitment

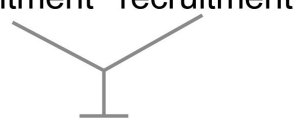

Anaphase onset 